\title{
Numerical simulation of pulsating turbulent channel flow
}

\author{
Alberto Scottia) \\ Department of Marine Sciences, University of North Carolina, Chapel Hill, North Carolina 27599 \\ Ugo Piomelli \\ Department of Mechanical Engineering, University of Maryland, College Park, Maryland 20742
}

(Received 31 July 2000; accepted 8 February 2001)

\begin{abstract}
Direct and large-eddy simulations of the Navier-Stokes equations are used to study the pulsating flow in a channel. The cases examined span a wide range of frequencies of the driving pressure gradient, and encompass different physical behaviors, from the quasi-Stokes flow observed at high frequencies, to a quasisteady behavior at the lowest ones. The validity of the dynamic Smagorinsky model to study this kind of unsteady flow is established by a posteriori comparison with direct simulations and experimental data. It is shown that the fluctuations generated in the near-wall region by the unsteady pressure gradient do not propagate beyond a certain distance $l_{t}$ from the wall, which can be estimated quite accurately by a simple eddy viscosity argument. No substantial departure from the Stokes regime at very high frequency $\left(\omega^{+}\right.$as high as 0.1$)$ is observed. The time-dependent characteristics of the flow are examined in detail, as well as the topology of the coherent structures. (C) 2001 American Institute of Physics. [DOI: 10.1063/1.1359766]
\end{abstract}

\section{INTRODUCTION}

Inherent unsteadiness of the driving conditions characterizes many turbulent flows, both natural (e.g., the gravitywave induced bottom boundary layer, the blood flow in large arteries, the flow around swimming fish, etc.) and artificial (for instance, the flow in the intake of a combustion engine, the flow in certain heat exchangers, and so on). Despite their physical relevance, unsteady turbulent boundary layers (BLs) have received relatively little attention compared to steady ones. Although unsteadiness extends the parameter space in more than one direction, thus considerably complicating the picture, the characterization of unsteady boundary layers is crucial in many disciplines, for instance to study the physics of sediment transport in coastal waters, the biology of blood circulation, and so on. Moreover, as was pointed out by Sarpkaya, ${ }^{1}$ by looking at features that are common to steady and unsteady BLs, we may understand better the underlying physics of turbulent BLs altogether.

Unsteadiness can be periodic (either pulsating, if the temporal mean is nonzero, or oscillating) or aperiodic, as in the case of flow undergoing a sudden acceleration/ deceleration. Although it is tempting to draw analogies between these two cases, it must be remembered that a flow subject to a homogeneous, time-varying, pressure gradient is equivalent, via a transformation of variables, to a flow in which the time-varying component of the pressure is replaced by an equivalent oscillation in the boundary condition. The same is not true if the pressure gradient is inhomogeneous, such as in the case of a flow accelerating in response to a contraction in the channel. While in the latter case the pressure gradient can directly alter the structure of

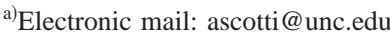

the flow, in the former it is the shear generated at the wall that affects the flow. ${ }^{2}$

The most studied periodic BLs geometry are flows over flat plates, pipes, and channels. The latter are the subject of this paper. Both oscillating and pulsating boundary layers admit a laminar solution, a trivial extension of Stokes' second problem, ${ }^{3-5}$ characterized by an oscillating part confined to a boundary layer whose thickness is $l_{s}=\sqrt{2 \nu / \omega}$, where $\nu$ is the kinematic viscosity, $\omega=2 \pi / T$ the frequency of oscillation, and $T$ its period. Of interest is the fact that both in the oscillating and in the pulsating flows (for sufficiently large values of the oscillating component of the velocity) the flow near the boundary reverses during part of the cycle without detachment of the boundary layer itself.

The literature on the subject of pulsating and oscillating flows is quite large. For a review, the reader is referred to Gündoğdu and Çarpinlioğlu. ${ }^{6,7}$ Here we just summarize the results more relevant to our investigation.

The purely oscillating case, which has been studied using measurements, ${ }^{8-12}$ flow visualizations, ${ }^{1}$ direct numerical simulations, ${ }^{13,14}$ and theoretical models ${ }^{15}$ is quite well understood. The relevant parameter is the Reynolds number based on the Stokes thickness $\operatorname{Re}_{s}=U_{o} l_{s} / \nu$, where $U_{o}$ is the oscillating mean-velocity component. According to Hino et al. ${ }^{9}$ the oscillating flow remains stable for $\operatorname{Re}_{s}<400$; for $400<\operatorname{Re}_{s}<800$ the flow undergoes a transition in which it cycles between laminar and turbulent states over a period; finally, it becomes fully turbulent for $\operatorname{Re}_{s}>800$. Sarpkaya ${ }^{1}$ observed that the flow varies from a featureless regime $\left(\operatorname{Re}_{s}<400\right.$, laminar) to a regime $\left(\operatorname{Re}_{s}>800\right)$ in which the population of hairpin vortices is quite different from the one observed in the steady case.

In the pulsating case, the focus is on understanding the interaction between the mean and the oscillating parts. Each ensemble-averaged quantity (indicated in the following by 
TABLE I. Parameters used in the experiments cited.

\begin{tabular}{llccc}
\hline \hline \multicolumn{1}{c}{ Group } & Geometry & $\omega^{+}$ & \multicolumn{1}{c}{$\operatorname{Re}$} & $a_{\text {uc }}$ \\
\hline Iowa $^{\mathrm{a}}$ & Pipe & $0.00079-0.0057$ & 25000 & $0.15-0.64$ \\
Illinois $^{\mathrm{b}}$ & Pipe & $0.21-0.0075$ & $7500-35000$ & $0.044-0.3$ \\
Grenoble $^{\mathrm{c}}$ & Channel & $0.001-0.25$ & $5000-10000$ & $0.14-0.64$ \\
Stanford $^{\mathrm{d}}$ & Flat plate & $0.0165-0.00077$ & 27000 & 0.15 \\
\hline \hline
\end{tabular}

${ }^{\mathrm{a}}$ References 20 and 21 .

${ }^{\mathrm{b}}$ References 22-24.

${ }^{\mathrm{c}}$ References 2, 17-19.

${ }^{\mathrm{d}}$ References 25 and 26 .

angular brackets, \langle\rangle$)$ can be seen as the sum of a time-mean and a periodic part. The flow is described by three parameters: (1) the mean flow (assumed to be nonzero and turbulent) defines a mean friction velocity $u_{\tau}$; (2) the forcing frequency in wall units, $\omega^{+}=\omega \nu / u_{\tau}^{2} \equiv 2 / l_{s}^{+2}$; (3) the ratio between oscillating and mean centerline (or free-stream for unbounded flows) velocity, $a_{\mathrm{uc}} \cdot{ }^{16}$ When $a_{\mathrm{uc}}<1$ (currentdominated flow), experiments ${ }^{17-19}$ show that the flow is largely controlled by $\omega^{+}$. Since this case is prevalent in the ocean, ${ }^{15}$ we will concentrate on this regime.

Several investigators have explored experimentally the current-dominated flow. We focus here on experiments performed by four experimental groups on pulsating flows during the last 20 years: They are the Iowa group, ${ }^{20,21}$ the Illinois group, ${ }^{22-24}$ the Stanford group, ${ }^{25,26}$ and the Grenoble group. ${ }^{2,17-19}$ The parameter space investigated by each group is summarized in Table I and Fig. 1. In Fig. 1, in order to classify the flows based on the driving frequency and the mean Reynolds number (as we have seen, the flows are only weakly controlled by the amplitude of the oscillation, provided $a_{\mathrm{uc}}<1$ ) we introduce the concept of turbulent Stokes length $l_{t}^{+}$, which defines how far vorticity waves generated

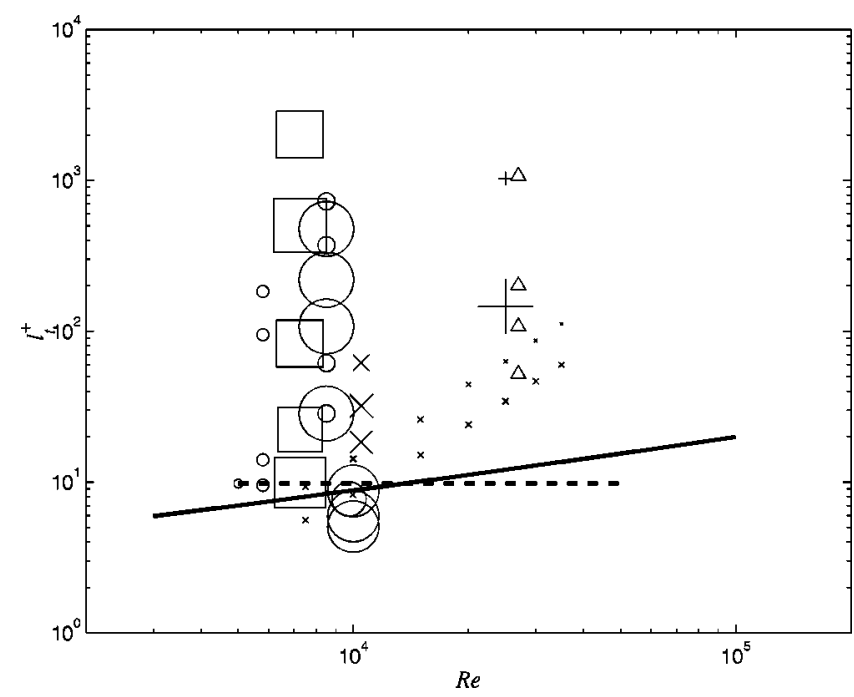

FIG. 1. Classification of unsteady pulsating flows based on mean Reynolds number, penetration length and amplitude of oscillation. The size of the symbols is proportional to $a_{\mathrm{uc}}$ for each experiment. ( $\left.\bigcirc\right)$ Grenoble group (Refs. 2, 17-19); (+) Iowa group (Refs. 20 and 21); (×) Illinois group (Refs. 22-24); ( $\triangle$ ) Stanford group (Refs. 25 and 26); ( $\square$ ) present calculations; (---) penetration length with $\omega^{+}$given by the bursting frequency reported by Kim and Spalart (Ref. 29); (-) penetration length with $\omega^{+}$given by the empirical formula by Demare et al. (Ref. 30). at the wall penetrate into the flow. ${ }^{27}$ Assuming that the effect of turbulence can be captured, at least qualitatively, by a simple eddy viscosity $\nu_{t}$, and drawing an analogy to the laminar Stokes problem, the effect of the oscillation on the flow is confined to a layer of thickness $2 l_{t}$, where $l_{t}$ $=\left[2\left(\nu+\nu_{t}\right) / \omega\right]^{1 / 2}$ is a turbulent Stokes length obtained using the sum of the molecular and turbulent diffusivities. Taking for the eddy viscosity $\nu_{t}=\kappa u_{\tau} l_{t}$, where $\kappa$ is the von Kármán constant, it follows that

$$
l_{t}^{+}=l_{s}^{+}\left[\left(\frac{\kappa l_{s}^{+}}{2}\right)+\sqrt{1+\left(\frac{\kappa l_{s}^{+}}{2}\right)^{2}}\right] .
$$

At high frequencies $\left(\kappa l_{s}^{+} / 2 \ll 1\right)$ the turbulent penetration length is $l_{t}^{+} \simeq l_{s}^{+}$, while at lower frequencies $l_{t}^{+} \simeq \kappa\left(l_{s}^{+}\right)^{2}$. A fluctuation of a given frequency propagates away from the generation area (while being attenuated) at a speed $c^{+}$ $\sim \omega^{+} l_{t}^{+}$(see the Stokes solution). At large values of $l_{s}^{+}$, we have from Eq. (1) $l_{t}^{+} \simeq \kappa l_{s}^{+2}$, which implies that $c^{+}$is constant and equal to $2 \kappa\left(\right.$ since $\left.\omega^{+}=2 / l_{s}^{+2}\right)$. Notice that the wavelength $\lambda^{+}=2 \pi l_{t}^{+}$is entirely contained within the penetration length. Except in the very high-frequency limit (where $l_{t}^{+} \simeq l_{s}^{+}$), the time required for a pulse to travel the half-width of the channel (or radius of the pipe) $h^{+}$relative to the period of the forcing is of the order $h^{+} \omega^{+} / c^{+}$ $=h^{+} / l_{t}^{+}$, so that $l_{t}^{+}$measures both how far and how quickly the pulse travels. When the period of the oscillation is large $\left(\omega^{+} \rightarrow 0\right.$, quasisteady regime), so that $l_{t}^{+} \gg h^{+}$, the turbulence has time to relax to the local (in time) equilibrium. ${ }^{19}$ In this case, the phase of all turbulent quantities becomes independent from $z^{+}$(the distance from the wall) and the flow is not different from a steady BL, provided that the instantaneous values for the driving forcing are used. It is difficult to pinpoint precisely where this regimes begins, and it is likely that the threshold depends on $a_{\mathrm{uc}}$.

As the frequency is increased and $l_{t}^{+}$becomes of the order of $h^{+}$, we enter the low-frequency regime, and several interesting phenomena occur: As one moves from the core toward the inner region of the flow, quantities such as production and dissipation become out of phase with respect to each other. The entire flow is affected by the unsteadiness. If the amplitude is large enough, relaminarization can occur, and the acceleration and deceleration phases are not symmetrical, as the production of turbulent kinetic energy is inhibited during the acceleration.

Further increasing the frequency leads us into the intermediate-frequency range. In this regime, $2 l_{t}^{+}<h^{+}$, so that there exists a region around the center of the channel or pipe where turbulence is frozen and simply advected as a plug flow. Within the region $z^{+}<2 l_{t}^{+}$, on the other hand, turbulent quantities are out of phase. A puzzling feature of this regime concerns the amplitude of the oscillating wall shear as a function of the forcing frequency. In the laminar case, the amplitude of the oscillating stress is proportional to $l_{s}^{-1} \simeq \sqrt{\omega}$; that is, the stress decreases as the frequency is lowered. At frequencies approaching the quasisteady regime the turbulent flow is in quasiequilibrium, and the observed stress becomes frequency independent. Thus, the stress scaled by its laminar value is greater than one. As the forcing 
frequency increases [see Fig. 3(a) in Tardu and Binder ${ }^{17}$ ] the amplitude of the oscillating part of the wall shear stress, scaled with its laminar Stokes prediction, decreases. This in agreement with the assumption that at very high frequencies the oscillating solution tends toward the laminar Stokes solution. However, when $0.005<\omega^{+}<0.02,\left(20<l_{t}^{+}<100\right)$ the scaled amplitude is found to be lower than 1 , which is somewhat paradoxical (it implies that the shear is being reduced by turbulence). Note that measurements close to the wall in this regime are difficult. The results of the Grenoble group support this picture, while the Illinois group results are inconclusive (Finnicum and Hanratty ${ }^{23}$ do not report values substantially lower than 1, while Mao and Hanratty ${ }^{22}$ do).

At high driving frequencies $\left(0.02<\omega^{+}<0.04,10<l_{t}^{+}\right.$ $<20)$ the Stokes layer remains confined within the viscous sublayer, so that one would expect the oscillating part to decouple from the turbulent mean. In this case, the amplitude of the modulation of turbulent quantities should decrease toward zero, while the amplitude $A_{\tau}$ and phase $\phi_{\tau}$ of the shear at the wall should relax toward the Stokes laminar values, respectively, $A_{\tau}^{\mathrm{St}}=\sqrt{2} a_{\mathrm{uc}} U_{\text {mean }} / l_{s}$ and $\phi_{\tau}^{\mathrm{St}}=\pi / 4$. This is indeed the case, but only if the driving frequency $\omega^{+}<0.04$, above which we enter in the so-called very high-frequency regime. In this range of parameters, the driving frequency becomes comparable with the bursting frequency and some experiments report (with considerable scatter) a deviation from the Stokes laminar regime: This means a relative increase of the modulation of fluctuation in the wall stress, and a reduced phase lead of the wall stress relative to the centerline velocity. This has been explained as a possible resonance of the driving conditions with the frequencies characteristic of the coherent structure dynamics in the inner layer. The Illinois and Grenoble groups explored this regime, and the agreement is only qualitative (see, for instance, Fig. 3 in Tardu and Binder ${ }^{17}$ ). On the other hand, Hwang and Brereton $^{28}$ studied the high- and very high-frequency limit in a pipe at $\operatorname{Re} \simeq 10^{4}$ and found no evidence of resonance. Note that the values reported in the literature for the bursting frequency as a function of the Reynolds number are affected by significant scatter, depending on the technique used to measure it. As an example, in Fig. 1 we have used the value reported by Kim and Spalart, ${ }^{29}$ which was deduced from direct numerical simulations (DNS) of boundary layer flows, and the experimental value reported by Demare et al. ${ }^{30}$ are shown. Other authors have reported lower frequencies. In particular, the experiments of Luchik and Tiedermann ${ }^{31}$ (who measured the bursting frequency using a variety of techniques in the range $\operatorname{Re}=8000-18000$ ) show that the frequency increases by almost a factor 2 as the Reynolds number goes from 8500 to 9500 , and becomes independent of the Reynolds number (when normalized using wall units) at larger values. Unfortunately, the majority of experiments in the very high-frequency regime were performed in this range of Reynolds numbers, so that similar values of $\omega^{+}$could fall into the high- or the very high-frequency regime in different experiments. Also, it is possible that the geometry of the experiment (channel versus pipe versus flat plate boundary layer) might affect the bursting frequency. ${ }^{32}$

There is no doubt that the presence of the mean flow affects the oscillating part in several ways, such as changing the phase structure and thickening the Stokes layer. The effects of the oscillating part on the mean flow are more controversial, and the experimental literature is divided on this issue. The Stanford group reports a substantial insensitivity of the mean velocity (which collapses on the usual law of the wall $U^{+}=5.5+1 / \kappa \log y^{+}$) and streamwise fluctuations. The data of the Grenoble group are less clear. The mean velocity collapse (with a non-negligible scatter) on a curve which is steeper than the usual law of the wall. The mean spanwise turbulent fluctuations increase with decreasing frequency, and only in the high-frequency regime agree with data from steady flow. Recently, Lodahl et al. ${ }^{12}$ undertook a comprehensive experiment covering a large cross section of the parameter space. They find that in order to have a significant modification in the mean properties, it is necessary that the oscillation of the free stream be larger than the mean (wavedominated case). In this case, the turbulence resembles the purely oscillating case, with a reduction in the mean stress at the wall if $\operatorname{Re}_{s}$ is in the laminar or transitional regime (relative to a purely oscillating case), or an increase in the stress if $\mathrm{Re}_{s}$ is sufficiently large. The explanation they propose is that, when $\operatorname{Re}_{s}$ is small and the flow is wave-dominated, the flow relaminarizes.

Turbulence models for pulsating boundary layers have been considered by several authors. ${ }^{33,34,6,7}$ In the majority of cases, they are extensions of steady eddy-viscosity closures, with different recipes to compute the eddy viscosity, the implicit assumption being that the turbulence is in quasiequilibrium. Based on the available experimental evidence, this is surely not the case unless the driving frequency is very small. One notable exception is the model proposed by Mankbadi and Liu, ${ }^{35}$ which is based on rapid distortion theory and it is a true unsteady model. The model of Mankbadi and $\mathrm{Liu}^{35}$ captures some of the features when the frequency approaches the quasisteady limit, but fails to reproduce the regime at higher frequencies. Mao and Hanratty ${ }^{22}$ have proposed a model that reproduces qualitatively the turbulentinduced reduction in amplitude described previously, based on the premise that the main effect of the oscillating pressure gradient is to change the height of the viscous layer. A relaxation relation is used to compute the height, which is hence out of phase with the outer layer. The eddy viscosity is computed using Reichardt's ${ }^{36}$ formula for the core region, while for the near-wall the mixing length approach with van Driest damping ${ }^{37}$ is used. The damping length is assumed to be proportional to the height of the wall layer. By their own admission, the model has very little physical justification. It also depends on constants that have to be fine-tuned. Nonetheless, it points to the fact that the heaving of the wall layer plays an important role.

Recently, Scotti and Piomelli ${ }^{38}$ tested various turbulence models for the unsteady Reynolds-averaged Navier-Stokes equations using the database described in this paper. They found that although all the models tested predicted the correct trends, they significantly overestimated the Reynolds stresses. The turbulent kinetic energy and the dissipation were also significantly overestimated.

Given the tremendous contribution that numerical simu- 
lations (both direct and large-eddy simulations) have given to our understanding of steady wall-bounded flows, ${ }^{39-41}$ it is surprising to find that only a handful of such studies is published on the topic at hand. In addition to the work by Spalart and Baldwin, ${ }^{13}$ which looked at the transition problem in the purely oscillating case, a recent paper by Hsu et al. ${ }^{42}$ compares a large-eddy simulation (LES) that uses the dynamic model $^{43}$ with Reynolds-averaged Navier-Stokes (RANS) calculations performed with the Saffman ${ }^{44}$ model, again for an oscillating flow over a plate. Yakhot et al. ${ }^{45}$ reported results concerning a laminar oscillating flow in a square duct, but were mainly concerned with the numerical aspects of the problem, and the effects of the aspect ratio of the duct. Tardu and Binder ${ }^{17}$ quote a DNS performed by Rida and Dan $\mathrm{Tran}^{46}$ in the high and very high frequency regimes.

As already recognized by Binder et al., ${ }^{19}$ there are no special technical difficulties in performing DNS of pulsating flows. The practical obstacle is represented by the long integration time required to achieve convergence, which increases as the $\mathrm{Re}_{s}^{2}$. While LES could provide substantial advantages, since its reduced cost allows an extensive study of the parameter space at a fraction of the cost of DNS, the oscillating nature of the forcing is felt by the small scales, as pointed out by Binder et al. ${ }^{19}$ so that before trusting the outcome of a large-eddy simulation based on standard closures, a careful, a posteriori comparison with DNS and experiments has to be done.

The aim of this paper is twofold. First, we want to show that a LES based on the dynamic Smagorinsky model is suited to tackle unsteady problems. Our main objective, however, is to study the pulsating BL over the whole frequency range. We focus on the current-dominated case for several reasons: First, as explained previously, good experimental data sets exist against which our experiment can be compared; while numerical simulations without mean flow have been reported, there does not seem to be any extensive study published in the current-dominated case; such an investigation can provide crucial answers to some of the questions that have been raised previously, such as the magnitude of the reduction of the stress in the intermediate region, possible influences on the mean properties of the modulation of the viscous sublayer and the topology of the coherent structures. Finally, this study intends to provide reference data to be used to test currently used unsteady Reynolds-averaged Navier-Stokes models ${ }^{38}$ as well as to gain information to be used to study the sediment transport in the wave boundary layer in relatively deep water, where currents are generally larger than the velocities induced by the waves at the surface (see Madsen and Grant ${ }^{15}$ ).

In the following, the governing equations and the numerical scheme will be presented. Then, Sec. III will be devoted to the validation of the LES, by comparison with DNS results and experimental data. Once the accuracy of LES is established, we will consider the physical aspects of the flow in Sec. IV. Some concluding remarks will follow.

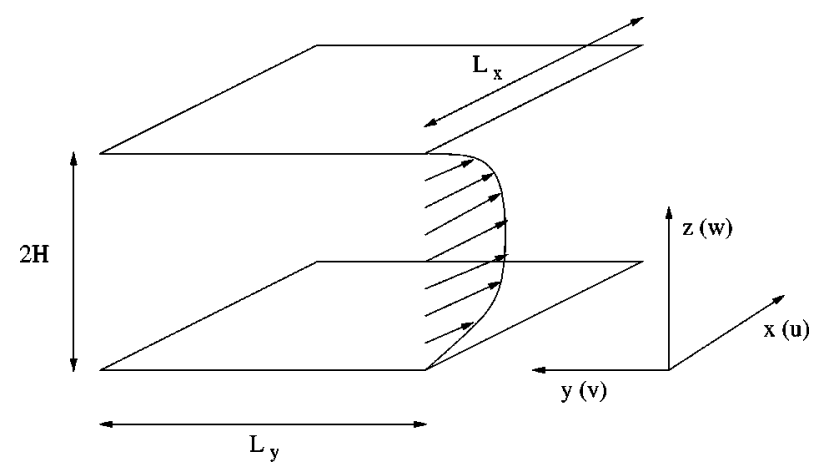

FIG. 2. Sketch of the computational domain and definition of the coordinates.

\section{PROBLEM FORMULATION}

\section{A. Governing equations and numerical method}

In this investigation either the Navier-Stokes equations

$$
\frac{\partial u_{i}}{\partial t}+\frac{\partial}{\partial x_{j}}\left(u_{i} u_{j}\right)=-\frac{\partial P}{\partial x_{i}}+\nu \frac{\partial^{2} u_{i}}{\partial x_{j} \partial x_{j}}, \quad \frac{\partial u_{i}}{\partial x_{i}}=0,
$$

or their filtered counterparts

$$
\frac{\partial \widetilde{u}_{i}}{\partial t}+\frac{\partial}{\partial x_{j}}\left(\widetilde{u}_{i} \widetilde{u}_{j}\right)=-\frac{\partial \widetilde{P}}{\partial x_{i}}+\nu \frac{\partial^{2} \widetilde{u}_{i}}{\partial x_{j} \partial x_{j}}-\frac{\partial \tau_{i j}}{\partial x_{j}}, \quad \frac{\partial \widetilde{u}_{i}}{\partial x_{i}}=0,
$$

where a tilde denotes a filtered variable, and

$$
\tau_{i j}=\left(\widetilde{u_{i} u_{j}}-\tilde{u}_{i} \tilde{u}_{j}\right)
$$

are solved. Here $(x, y, z)$ or $\left(x_{1}, x_{2}, x_{3}\right)$ stand for the streamwise, spanwise, and vertical coordinates, with $(u, v, w)$ or $\left(u_{1}, u_{2}, u_{3}\right)$ the respective velocities (see Fig. 2). The geometry used is a channel of height $2 H$, and width $L_{x}$ and $L_{y}$, respectively. Periodic boundary conditions are used in $x$ and $y$, and no-slip conditions at the upper and lower boundaries. The flow is forced by an external pressure field given by

$$
P_{f}(x, t)=\Delta P_{o}[1+\alpha \cos (\omega t)] x / L_{x} .
$$

The total pressure $\widetilde{P}$ is equal to the sum of the forcing pressure $P_{f}$, and the mechanical pressure $p(x, y, z, t)$ required to keep the flow divergence free. For the LES the subgrid-scale stresses ${\widetilde{u_{i}}}_{i}-\widetilde{u}_{i} \widetilde{u}_{j}$ were modeled using the dynamic eddyviscosity model. ${ }^{43,47}$ The present implementation of the model is described in detail in Piomelli ${ }^{48}$ and will not be repeated here.

The equations are solved using a pseudospectral code $^{48-50}$ that uses Fourier expansions in the homogeneous directions and Chebychev collocation in the vertical one, which was modified to run on parallel computers. ${ }^{51}$ Five computational grids were considered. Table II lists the properties of the grids used. Two DNS calculations were carried out; only a very small difference between their results could be observed, indicating that grid invariance of the solution had been achieved. In the following only the results of the finer calculation, DNS1, will be shown. The LES grid with the finest vertical resolution (LES3) was used to study the flow at the highest driving frequency, to assure that the large gradient at the wall was well-resolved. 
TABLE II. Grids employed. Wall units are defined in Sec. II B.

\begin{tabular}{lrrlll}
\hline \hline Grid & \multicolumn{1}{c}{$\Delta x^{+}$} & \multicolumn{1}{c}{$\Delta y^{+}$} & \multicolumn{1}{c}{$L_{x}$} & \multicolumn{1}{c}{$L_{y}$} & \multicolumn{1}{c}{ Mesh } \\
\hline DNS1 & 25.6 & 5.7 & $3 \pi H$ & $\pi H$ & $128 \times 192 \times 129$ \\
DNS2 & 34.3 & 11.4 & $4 \pi H$ & $4 \pi H / 3$ & $128 \times 128 \times 97$ \\
LES1 & 137.3 & 45.8 & $4 \pi H$ & $4 \pi H / 3$ & $32 \times 32 \times 49$ \\
LES2 & 51.5 & 17.1 & $3 \pi H$ & $\pi H$ & $64 \times 64 \times 65$ \\
LES3 & 51.5 & 17.1 & $3 \pi H$ & $\pi H$ & $64 \times 64 \times 97$ \\
\hline \hline
\end{tabular}

\section{B. Parameter space}

As mentioned previously, in the current dominated case the experiments show that the flow is more sensitive to changes in $l_{s}^{+}$than to changes in $a_{\mathrm{uc}}$; in all of our experiments we have kept $\Delta P_{o}$ constant (which fixes the mean friction velocity, see the following below) and we have changed $\omega$ and $\alpha$ to achieve the desired value for $a_{\mathrm{uc}}$ and $l_{s}^{+}$. Wall units were obtained using the time-mean friction velocity, which is a function of the mean pressure gradient: $u_{\tau}$ $=\sqrt{H \Delta P_{o} / 2 \rho L_{x}}$. By setting $\Delta P_{o} / L_{x}=1.0 \times 10^{-4}$ the Reynolds number based on the mean friction velocity and channel half-height, $\operatorname{Re}_{\tau}=u_{\tau} H / \nu$, was equal to 350 , while the one based on the mean centerline velocity $\operatorname{Re}_{H}=U_{c} H / \nu$ had a weak dependence on $l_{s}^{+}$, but was approximatively equal to 7250. We conducted calculations with values of $l_{s}^{+}$, ranging from 4.4 to 70. In all calculations $a_{\text {uc }}$ was close to 0.7. Table III reports the relevant parameters for each run.

\section{Data reduction}

In unsteady flows such as the present one several types of averaging operators can be defined. First, a quantity can be averaged over planes of homogeneity ( $x y$ planes) and time; such quantity will be denoted by a bar or by a capital letter:

$\bar{f}(z)=F(z)=\frac{1}{T_{\text {tot }} L_{x} L_{y}} \int_{0}^{T_{\text {tot }}} \int_{0}^{L_{y}} \int_{0}^{L_{x}} f(x, y, z, t) d x d y d t$,

where $T_{\text {tot }}=N T$ is the total time of integration, and $N$ the total number of periods over which the equation have been integrated. Note that the over bar denoting the filtering operation will be dropped; it will be clear from the context whether the discussion refers to the total or the filtered velocity.

In addition to plane and time averaging, phase averaging is effective in extracting the coherent response of the system

TABLE III. Parameters, grids, and models used for the simulations.

\begin{tabular}{llllll}
\hline \hline & $\begin{array}{c}\text { Very high } \\
\text { frequency }\end{array}$ & $\begin{array}{c}\text { High } \\
\text { frequency }\end{array}$ & $\begin{array}{c}\text { Medium } \\
\text { frequency }\end{array}$ & $\begin{array}{c}\text { Low } \\
\text { frequency }\end{array}$ & Quasisteady \\
\hline$\omega^{+}$ & 0.1 & 0.04 & 0.01 & 0.0016 & 0.0004 \\
$\alpha \cdot \Delta P_{o} / L_{x}$ & 0.05 & 0.02 & 0.005 & 0.0008 & 0.0002 \\
$\mathrm{Re}_{s}$ & 63 & 100 & 200 & 500 & 1000 \\
$l_{s}^{+}$ & 4.4 & 7 & 14 & 35 & 70 \\
$a_{\text {uc }}$ & 0.75 & 0.66 & 0.70 & 0.79 & 0.71 \\
Grids & LES3 & DNS1, DNS2 & LES1 & LES1 & LES1 \\
& & LES1, LES2 & LES2 & LES2 & \\
\hline \hline
\end{tabular}

to the pulsating forcing. Experiments with pulsating flows have shown that the frequency spectra of most measured quantities show a peak at the forcing frequency, ${ }^{22,26}$ with no significant (coherent) response at lower frequencies. Hence, in the present study, we have phase averaged the quantities of interest over the period set by the forcing:

$$
\langle f\rangle(z, t)=\frac{1}{N L_{x} L_{y}} \sum_{n=1}^{N} \int_{0}^{L_{y}} \int_{0}^{L_{x}} f(x, y, z, t+n T) d x d y .
$$

Furthermore, the phase-averaged quantities can be considered as the superposition of a mean part, an oscillating component with period equal to the one of the driving frequency (i.e., the first Fourier mode in the expansion of the quantity under consideration), and a part that includes the contribution at all the other frequencies generated by the nonlinear interactions in the system:

$$
\langle f\rangle=F(z)+A_{f}(z) \cos \left[\omega t+\phi_{f}(z)\right]+\varepsilon(z, t),
$$

where $A_{f}(z)$ and $\phi_{f}(z)$ are chosen to minimize the $L_{2}$ norm of $\varepsilon,\|\varepsilon(z, t)\|_{2}$. In analogy with electric currents, we call $F$ the dc component, $A_{f}$ the ac component, and $\phi_{f}$ the phase. In the following, a prime and a double prime will be used to denote fluctuations with respect to the long-time and to the phase averages:

$f^{\prime}\left(x_{i}, t\right)=f\left(x_{i}, t\right)-F(z), \quad f^{\prime \prime}\left(x_{i}, t\right)=f\left(x_{i}, t\right)-\langle f\rangle(z, t)$.

\section{LES VALIDATION}

The dynamic eddy-viscosity model has been tested extensively on steady, wall-bounded flows, both transitional ${ }^{52}$ and turbulent. ${ }^{48}$ This model has shown itself capable to provide a good approximation of the large-scale physics, and has been applied to a variety of steady problems. To the best of our knowledge, however, its validity has never been rigorously tested in situations characterized by externally imposed unsteadiness, with the exception of a recent paper by Hsu et al. ${ }^{42}$ in which it was compared to a model for the Reynolds-averaged Navier-Stokes equations. In this section we compare the results from the DNS at $l_{s}^{+}=7$ with the LES. The focus is on establishing the validity of using LES in oscillating flows, so the physical interpretation of the results will be postponed until Sec. IV.

Figure 3 shows the averaged streamwise velocity. In the following, the velocities are made dimensionless by $u_{\tau}$, unless otherwise noted. The agreement is fairly good even for the LES on the coarse grid. The actual value of $u_{\tau}$ obtained from the calculations allows us to estimate the convergence of the statistical sample, since, for an infinitely long sample, $u_{\tau} \rightarrow \sqrt{H \Delta P_{o} / 2 \rho L_{x}}$. In the present case, the difference between the actual $u_{\tau}$ and its asymptotic value is less than $2 \%$ for the LES. For the DNS, the convergence is not as good, the difference being approximately $5 \%$, but still satisfactory. The larger scatter in the DNS is caused by the smaller sample (the DNS was substantially more expensive than the LES calculations, and was run for a smaller number of cycles); the scatter is, in the worst case, comparable to the experimental error. 


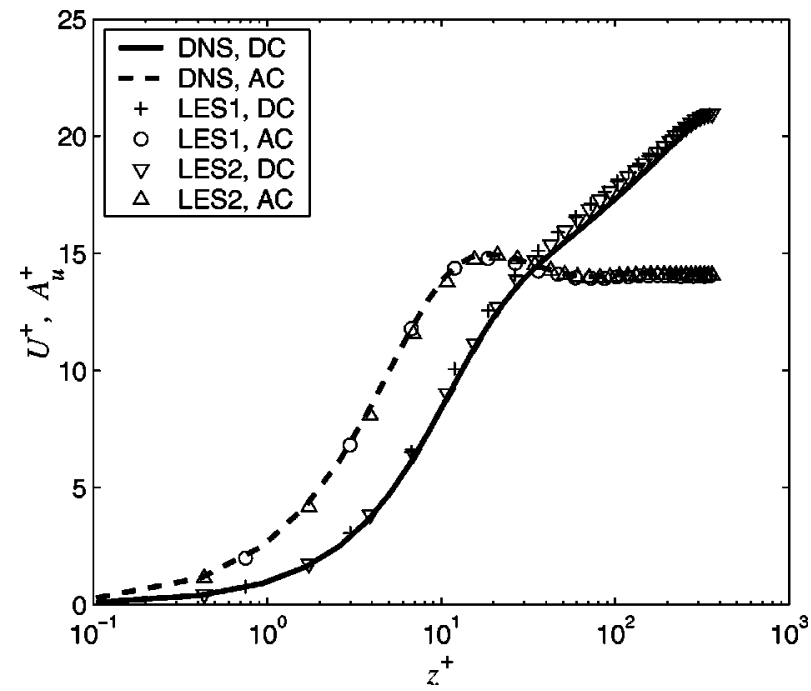

FIG. 3. dc and ac components of the streamwise velocity. $l_{s}^{+}=7$. dc component: (-) DNS; (+) LES1; $(\triangle)$ LES2. ac component; (---) DNS; $(\bigcirc)$ LES1; $(\triangle)$ LES2.

The time-averaged Reynolds stresses $R_{i j}=\overline{u_{i}^{\prime} u_{j}^{\prime}}$ and their ac components $A_{i j}$ are shown in Fig. 4. Again, the agreement is quite good, except for the wall-normal component, which is particularly affected by the small scales that are not resolved in the LES. The agreement for the oscillating part is better.

Another important benchmark for LES is how well the spectra are calculated. In Fig. 5 the turbulent kinetic energy
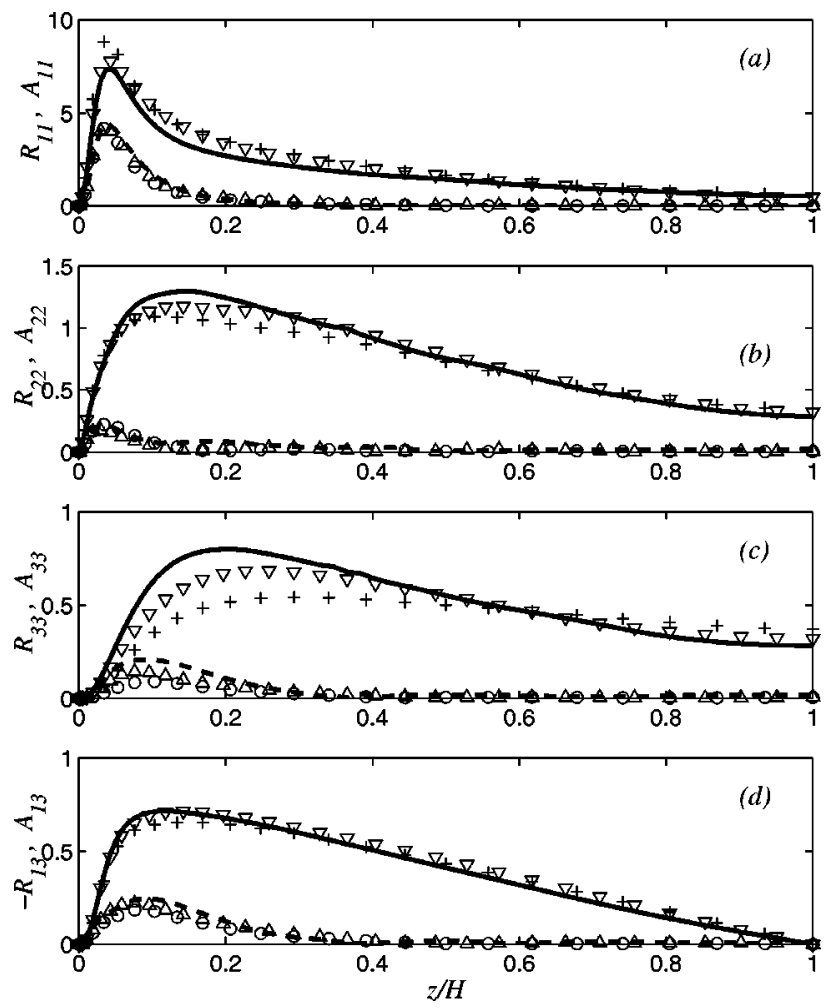

FIG. 4. dc and ac components of the Reynolds stresses. $l_{s}^{+}=7$. dc component: (-) DNS; (+) LES1; ( $\nabla)$ LES2. ac component: (---) DNS; (O) LES1; $(\triangle)$ LES2.

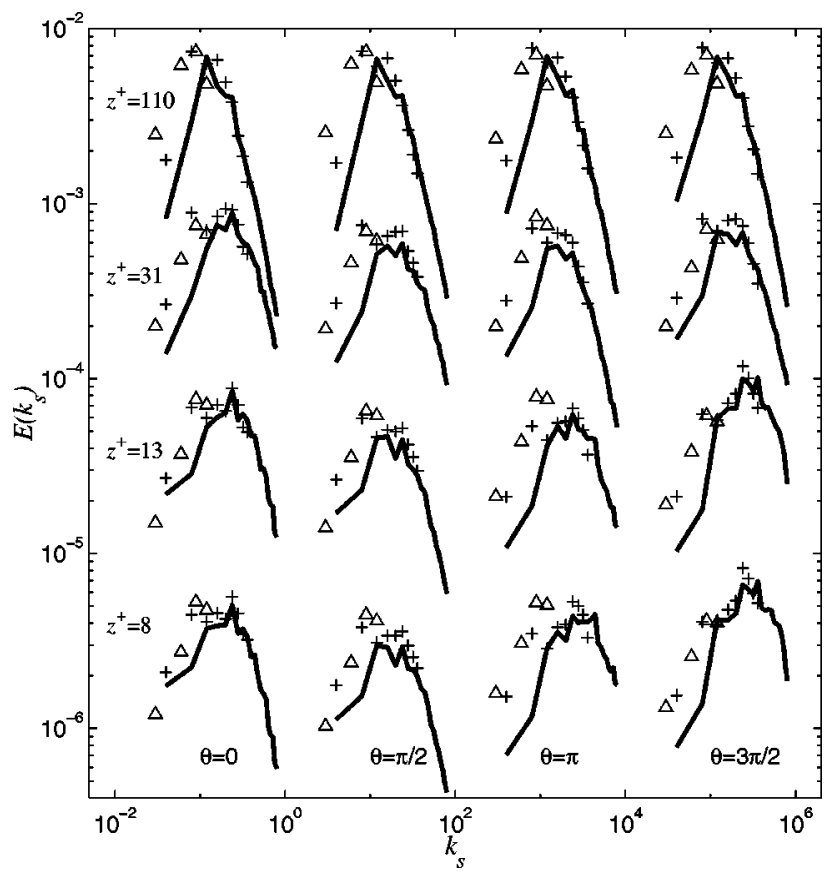

FIG. 5. 2-D spectra of turbulent kinetic energy during a wave cycle at (from top to bottom) $z^{+}=8,13,31$, and 110 , and (from left to right) $\theta=0, \pi / 2, \pi$, and $3 \pi / 2$. The spectra are offset with respect to each other by a factor of 10 in both the horizontal and vertical directions. (-) DNS; (+) LES2; $(\triangle)$ LES1.

spectra at four heights above the wall $\left(z^{+}=8,13,31\right.$, and $110)$ are shown at four equi-spaced times during a cycle. The spectra are integrated over cylindrical shells $k_{s}^{2}=k_{x}^{2}+k_{y}^{2}$. The two-dimensional (2-D) spectra are well reproduced both by LES1 and LES2. They show that for LES1 the cutoff lies well outside the inertial range, in the region in which the energy is still growing; it is somewhat surprising that LES1 is still able to capture qualitatively the main features of the flow. For LES2 the cutoff lies in the inertial range throughout the whole cycle. Note how the scale that marks the beginning of the inertial range oscillates during the wave cycle. This is especially true at $z^{+}=8$ and 13 .

In conclusion, for all the statistics computed (including those not shown here, such as the terms in the kinetic energy budget, the correlation coefficients, etc.) the LES gave fair to good agreement with the DNS data. When the cutoff scale lies in the inertial range, the results agree well quantitatively with the DNS. Qualitative agreement was observed even when the cutoff did not reach into the decaying region of the spectrum.

\section{RESULTS AND DISCUSSION}

\section{A. Turbulent statistics}

Having established the validity of the LES approach, we now turn to the more physical aspects of the flow. Figure 6 shows the time development of the centerline velocity $U_{\mathrm{cl}}$, wall stress $\tau_{w}$, and the maximum of the turbulent kinetic energy $K=\left\langle u_{i}^{\prime \prime} u_{i}^{\prime \prime}\right\rangle / 2$. To understand the main characteristics of the flow, consider the phase-averaged streamwise momentum equation: 


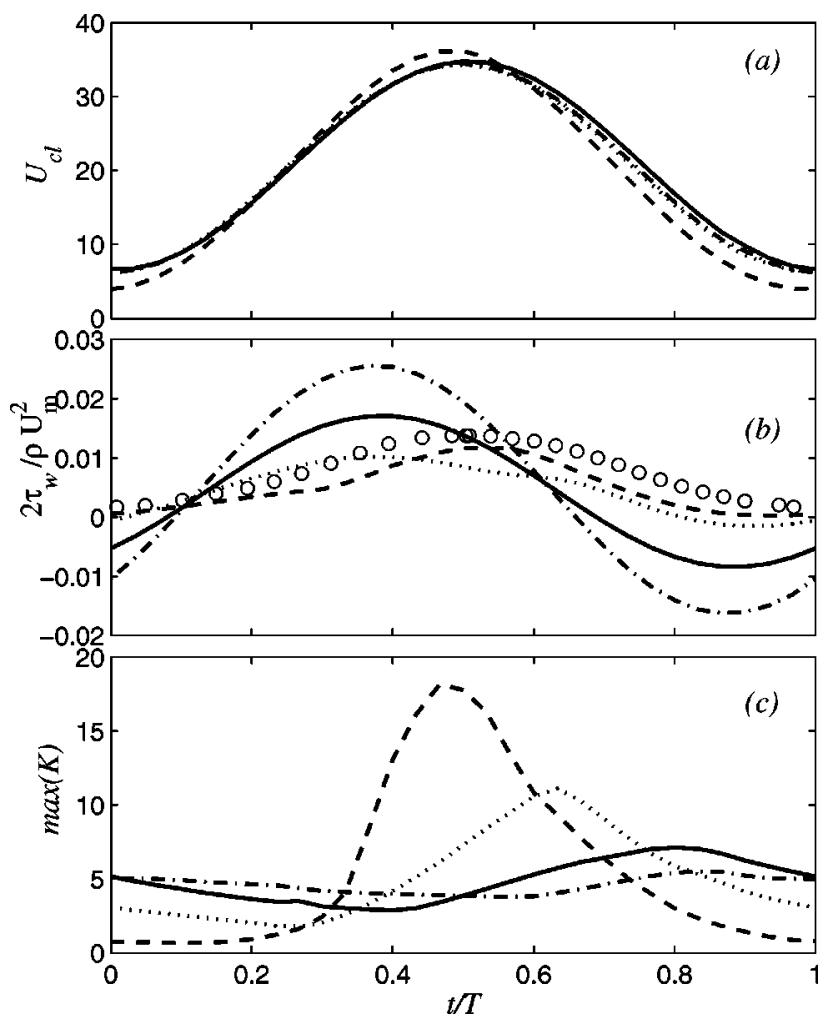

FIG. 6. Time development of (a) the centerline velocity $U_{\text {cl }}$; (b) the wall stress $2 \tau_{w} / \rho U_{m}^{2}$; (c) the peak value of the turbulent kinetic energy $K$ across the channel. (-.-) $l_{s}^{+}=4$; (-) $l_{s}^{+}=7 ;(\cdots) l_{s}^{+}=14$; (--) $l_{s}^{+}=35$; (○) experiments at $l_{s}^{+}=44$ (Ref. 20).

$$
\frac{\partial\langle u\rangle}{\partial t}=\frac{\partial}{\partial z}\left[-\left\langle u^{\prime} w^{\prime}\right\rangle+\frac{1}{\operatorname{Re}} \frac{\partial\langle u\rangle}{\partial z}\right]-\frac{\partial P_{f}}{\partial x},
$$

where lengths are made nondimensional by the channel halfheight $H$ and time by $H / u_{\tau}$ (the Reynolds number is, therefore, defined as $\left.\operatorname{Re}=H u_{\tau} / \nu\right)$. The oscillating component of the pressure field can be eliminated if we apply the following change of variables:

$$
\langle u\rangle \rightarrow\langle u\rangle+\int_{0}^{t} \frac{\partial P_{f o}\left(t^{\prime}\right)}{\partial x} d t^{\prime},
$$

with the boundary condition

$$
\langle u\rangle(0, t)=\int_{0}^{t} \frac{\partial P_{f o}\left(t^{\prime}\right)}{\partial x} d t^{\prime},
$$

where $P_{f o}$ is the unsteady component of the pressure. In the new variables the source of the oscillation is the boundary condition at the wall. Thus, an oscillating shear is set up at the wall, which propagates away from the wall. This, in turn, interacts with the steady turbulent field to produce an oscillating response in the turbulent quantities.

The scenario is thus as follows: The oscillation of the wall (i.e., the oscillating pressure) creates an oscillating shear which generates fluctuations in the turbulent quantities, both at the forcing frequencies and at higher harmonics. Only the fluctuations whose frequency is lower than a certain value (controlled by $\kappa l_{s}^{+} / 2 \gg 1$ ) leak out past the buffer, which acts as a low-pass filter.

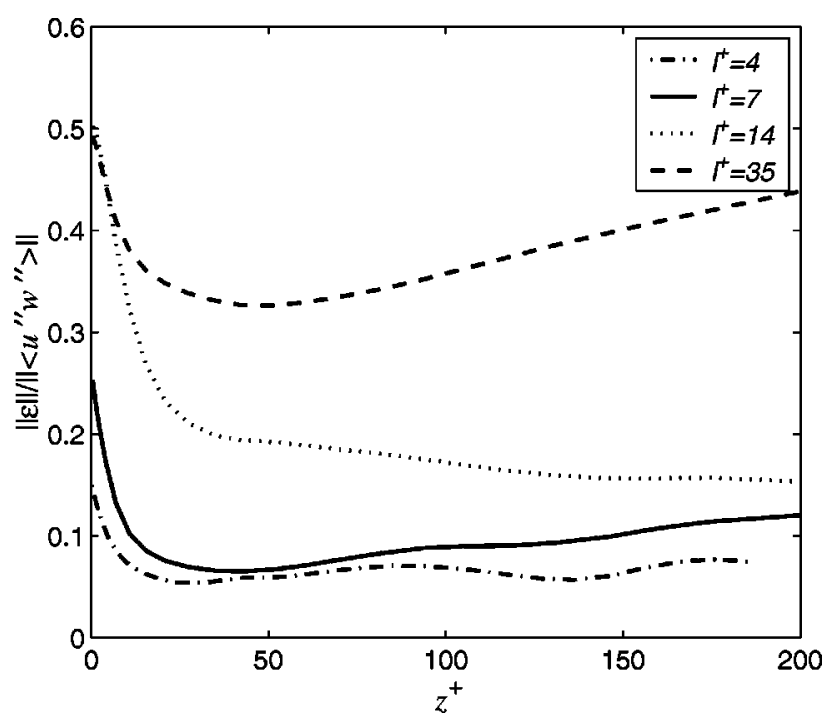

FIG. 7. Fraction of the variance of $\left\langle u^{\prime \prime} w^{\prime \prime}\right\rangle$ contained in frequencies higher than the fundamental.

At high frequencies $\left(l_{s}^{+}=4.4\right.$ and 7 , that is $l_{t}^{+}=10$ and 22 , respectively), the inner and outer layers are only weakly coupled, and we observe a sinusoidal behavior for $\tau_{w}$ and for the peak turbulent kinetic energy (that is the maximum value attained by the turbulent kinetic energy at a given time across the channel), shown in Figs. 6(b) and 6(c), respectively. The $45^{\circ}$ phase lag, typical of the laminar Stokes solution, can be observed for both quantities. For $l_{s}^{+}=14\left(l_{t}^{+}\right.$ $=82$ ), $\tau_{w}$ still exhibits a quasisinusoidal behavior, while the turbulent kinetic energy does not. Also note that the phase lead of the wall stress is now smaller than in the high frequency case. For $l_{s}^{+}=35\left(l_{t}^{+}=500\right)$ the behavior of both quantities is quite different from the high-frequency case.

This point is further illustrated in Fig. 7, which shows the contribution of the fundamental mode to the phaseaveraged Reynolds stress $\left\langle u^{\prime \prime} w^{\prime \prime}\right\rangle$. We define the error as

$$
\varepsilon(z, t)=\left\langle u^{\prime \prime} w^{\prime \prime}\right\rangle-\left[R_{13}+A_{13} \cos \left(\omega t+\phi_{11}\right)\right]
$$

and then consider its norm,

$$
\|\varepsilon\|=\left(\frac{1}{T} \int_{0}^{T} \varepsilon^{2} d t\right)^{1 / 2}
$$

normalized by the norm of $\left\langle u^{\prime \prime} w^{\prime \prime}\right\rangle$. Small values of $\|\varepsilon\|$ indicate sinusoidal behavior. The phase-averaged quantities progressively depart from a pure sinusoidal behavior as the frequency is lowered. This trend reverses somewhere above $l_{s}^{+}=35$, as the quasisteady limit is reached. Thus the lowfrequency regime differs from the quasisteady state in the amount of energy contained in oscillation at frequencies higher than the fundamental, with the low-frequency state displaying a stronger nonlinear response than the quasisteady one. At high frequencies our simulations confirm the established notion that the response of the system (at least in the averaged sense) is primarily at the forcing frequency. ${ }^{26}$ Higher harmonics, if present, are filtered out. The sinusoidal response, however, becomes more and more distorted as the frequency is lowered. 

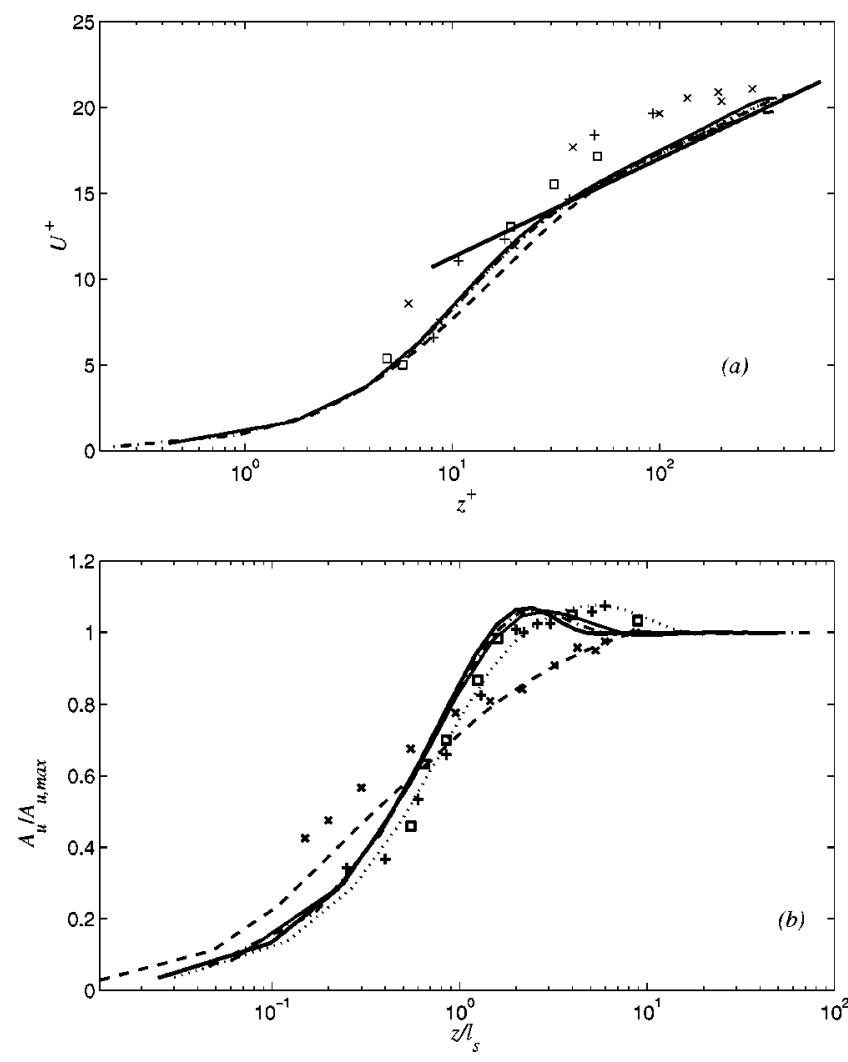

FIG. 8. Streamwise velocity profiles. (a) dc component; (b) ac component. Lines: LES; symbols: experiments (Ref. 18). (-.-) $l_{s}^{+}=4$; ( $\left.\square\right),(-) l_{s}^{+}$ $=7 ;(+)(\cdots) l_{s}^{+}=14 ;(\times)(--) l_{s}^{+}=35$. The thick solid lines indicate the logarithmic law of the wall in (a) and the Stokes solution in (b).

Figure 8 shows the dc and ac components of the streamwise velocity. The results collapse on the standard logarithmic layer for all frequencies examined. The experimental data ${ }^{18}$ are consistently higher than the present ones, and show a lower slope of the logarithmic layer. Data from the Stanford group, on the other hand, show a close agreement of $U^{+}$with the law of the wall at all frequencies considered. The presence of the dc component [Fig. 8(b)] begins to affect the ac part already at moderately high frequencies. Only when $l_{s}^{+}=4.4$ does the ac component match the Stokes solution. At $l_{s}^{+}=7$ the Stokes solution still applies in the viscous sublayer $\left(z^{+}<20\right)$, but the computed velocity exceeds it where the ac component of the Reynolds stress becomes significant $\left(20<z^{+}<60\right) . l_{s}^{+}=14$ represents an intermediate case: without a dc component the flow would be still laminar (the transition to a "preturbulent" state occurs around $\mathrm{Re}_{s}$ $=400$ in the DNS of oscillating boundary-layer flow by Spalart and Baldwin ${ }^{13}$ ). Here, the mean flow causes the upper part of the Stokes layer to be exposed to enhanced momentum transport. As a result, the ac part of the velocity still resembles the Stokes solution, albeit with an increased thickness, evidence of the increased mixing due to turbulence. At still larger values of $l_{s}^{+}$, the ac component becomes similar to a typical turbulent profile with a logarithmic layer. At this driving frequency the response of several turbulent quantities deviates significantly from a sinusoid, especially in the nearwall region. The agreement with the experimental data ${ }^{18}$ is, in this case, better.

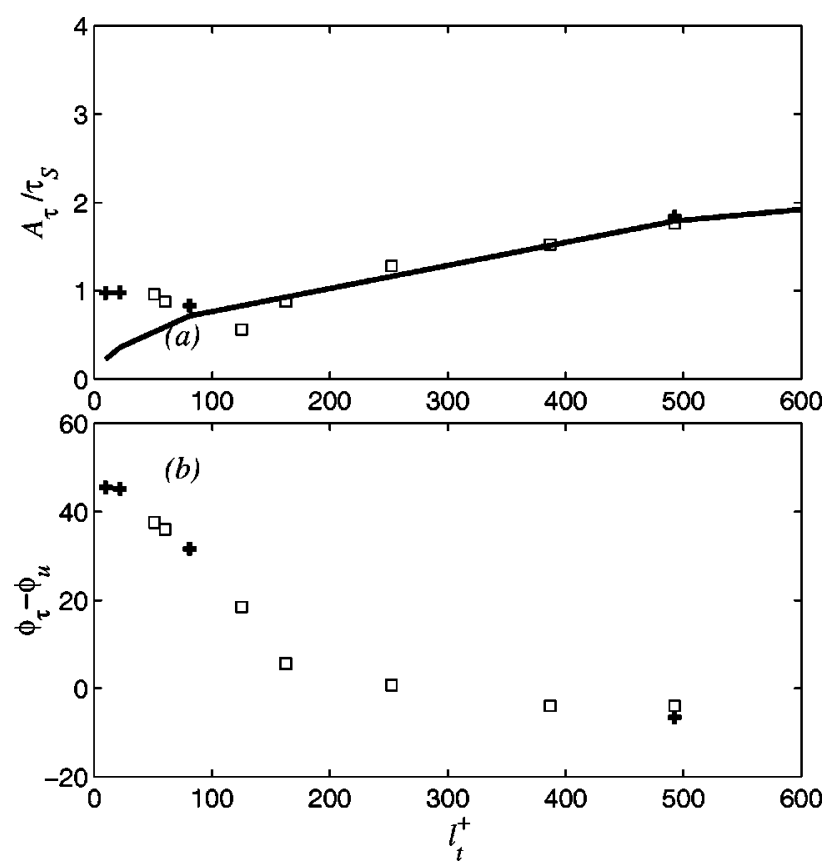

FIG. 9. ac component the wall stress. (a) Amplitude; (b) phase. (+) LES; $(\square)$ experiments (Ref. 18); (-) quasisteady solution.

In our calculations the dc wall-shear stress is fixed by the choice of $\Delta P_{o}$. In Fig. 9 we show the amplitude of the ac component normalized by the Stokes value $\tau_{s}=\sqrt{2} \nu U_{o} / l_{s}$. At low $l_{t}^{+}$, the stress is essentially identical to the Stokes value, as expected from the analysis of the momentum profiles. As $l_{t}^{+}$grows, the normalized amplitude first decreases and then increases to become significantly larger than the viscous value. The latter behavior is easy to understand if one considers that the flow tends toward a quasiequilibrium state, where the stress is controlled by the Blasius formula $\tau_{w}=0.048 \operatorname{Re}_{H}^{-1 / 4}\left(\rho U_{c}^{2} / 2\right)$. The solid line in Fig. 9 is the value computed assuming a quasisteady limit. ${ }^{18}$ The rather surprising fact that there is a range of values centered around $l_{t}^{+}=125$ for which the ac stress at the wall is smaller than the one predicted by the laminar theory has been observed in several experiments ${ }^{22,18,53}$ and many explanations have been proposed. They all hinge on the observation that as $l_{s}^{+}$increases, the oscillating flow is exposed to a turbulent viscosity that is both time and space varying. The net effect is that the oscillating velocity is similar to the Stokes layer but with a thicker boundary layer, which results in a smaller shear at the wall (recall that the laminar shear in the viscous Stokes is proportional to $\nu^{-1 / 2}$, that is, decreases with increasing viscosity). Our velocity profiles [Fig. 8(b)] and wall stress results support this explanation. When $l_{t}^{+} \simeq 150$ the stress at the wall becomes in phase with the outer flow.

The effect of the oscillation on the mean stress is less clear. Tardu et al. ${ }^{18}$ in whose experiments the mass flow rate was fixed, do not report any significant departure in the mean stress with and without oscillations. On the other hand, Lodahl et al. ${ }^{12}$ report a nontrivial dependence, with either a decrease in the mean stress or an increase, according to the relative strength of steady and oscillating forcing. Maintaining a fixed mass flow rate (as in the experiments) or constant 


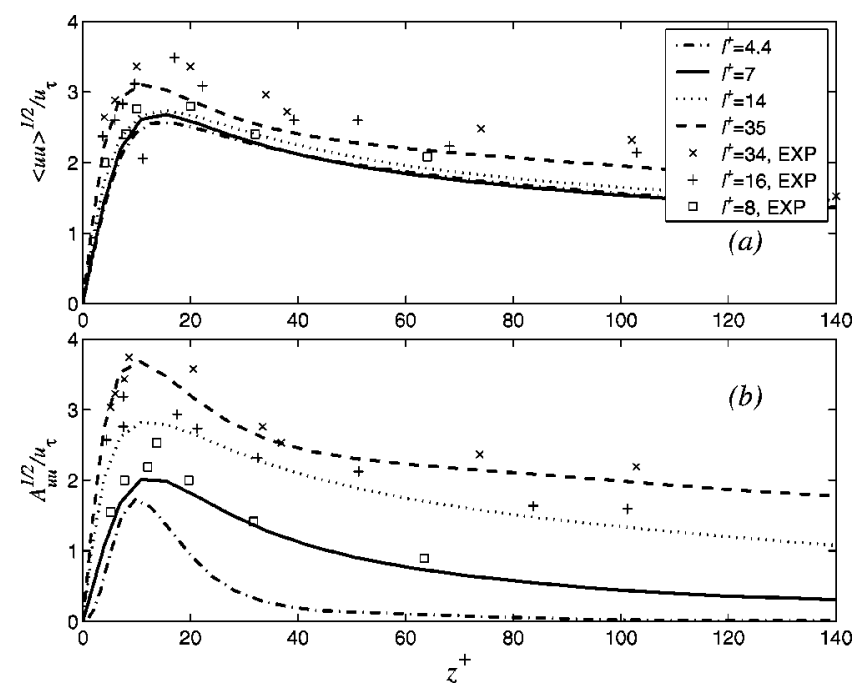

FIG. 10. dc (a) and ac (b) components of the streamwise turbulent fluctuations. Symbols are as in Fig. 8.

pressure gradient (as in the present calculations) is equivalent in steady flows; in the present case, however, the use of a constant mass flow rate allows $\mathrm{Re}_{\tau}$ to vary; the turbulent fluctuations may then be affected by changes in $\operatorname{Re}_{\tau}$. Maintaining $\operatorname{Re}_{\tau}$ constant, on the other hand, allows one to better isolate the effects of the unsteadiness on the turbulence. In our calculations the mean flow rate shows fluctuations of the order of $10 \%$, without a definite trend. In the very highfrequency case, our results agree with the finding of Finnicum and Hanratty ${ }^{23}$ that the oscillating part is given essentially by the Stokes solution, with a lead of $45^{\circ}$ in the shear at the wall, and at variance with the values reported by the Grenoble group, which show a lead of only about $20^{\circ}$. Mao and Hanratty ${ }^{24}$ have reported leads in excess of $60^{\circ}$ in the high- and very high-frequency regimes, when $a_{\mathrm{uc}}$ is large enough to ensure flow reversal near the wall, and a small but significant decrease in mean stress at the wall. However, the technique used to measure the stress (electrochemical probes) has not been tested extensively when flow reversal is present. $^{54}$

To validate our data set further we consider the statistics of the streamwise turbulent intensity, shown in Fig. 10, using the data from the Grenoble group as a reference. Similar to what was observed for the mean momentum, the agreement is quite good at all frequencies considered (there are no published data on the profile of $\left\langle u^{\prime \prime} u^{\prime \prime}\right\rangle$ at very high frequencies).

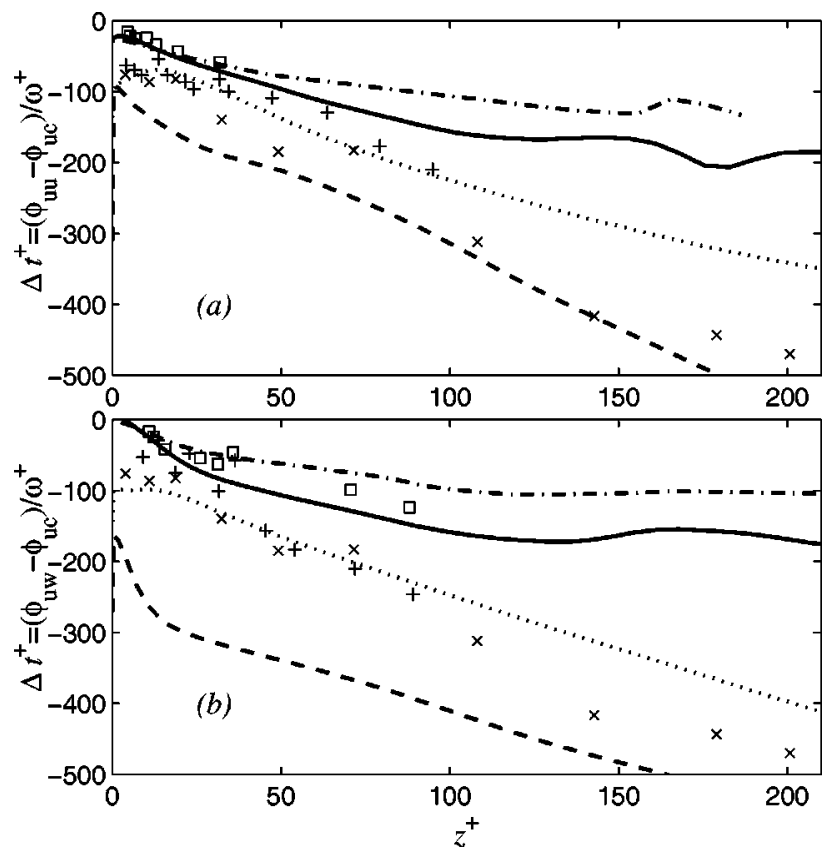

FIG. 11. Time lag (relative of centerline velocity) of streamline turbulent fluctuations (a) and turbulent Reynolds stress (b). Symbols are as in Fig. 8.

Regarding the dc component, shown in Fig. 10(a), a trend toward higher values as the driving frequency is lowered can be observed, although less evident than in the Grenoble data. The agreement with the experiments is better for the amplitude of the oscillation at the fundamental, shown in Fig. 10 (b). It is interesting to note that the amplitude of the ac part measured by the Grenoble group at low frequency is larger than the dc part. Since $\left\langle u^{\prime \prime} u^{\prime \prime}\right\rangle$ is a positive-definite quantity, it follows that a significant fraction of the total variance must be contained in higher harmonics, as we have discussed earlier regarding the time evolution of the peak kinetic energy. As for the mean streamwise velocity, the Stanford data do not show any significant trend as the frequency is lowered. We draw similar conclusions regarding the time lag (phase difference), shown in Fig. 11(a) for the streamwise-fluctuation variance and in Fig. 11(b) for the Reynolds shear stress.

The analysis of the turbulent statistics considered so far provides further confirmation that the modeling approach employed (LES) provides results that are consistent with the published literature. In Table IV we summarize the main findings concerning the controversial aspects highlighted in

TABLE IV. Results concerning mean properties, very high-frequency (VHF), and intermediate-frequency (IF) effects from the experiments considered and the present simulation.

\begin{tabular}{|c|c|c|c|}
\hline Experiments & Mean properties & VHF & IF \\
\hline Stanford & $\begin{array}{l}\text { Constant }\left\langle u^{\prime \prime} u^{\prime \prime}\right\rangle, \\
\text { law of wall }\end{array}$ & N.A. & N.A. \\
\hline Illinois & N.A. & Inconclusive & Inconclusive \\
\hline Grenoble & $\begin{array}{l}\text { Modified law of the wall, } \\
\left\langle u^{\prime \prime} u^{\prime \prime}\right\rangle \text { increases with } l_{s}^{+}\end{array}$ & Deviation from Stokes & Deviation from Stokes \\
\hline Iowa & Constant $\left\langle u^{\prime \prime} u^{\prime \prime}\right\rangle$ & N.A. & N.A. \\
\hline This study & $\begin{array}{l}\text { Constant }\left\langle u^{\prime \prime} u^{\prime \prime}\right\rangle \text {, } \\
\text { law of wall }\end{array}$ & Stokes & Stokes \\
\hline
\end{tabular}



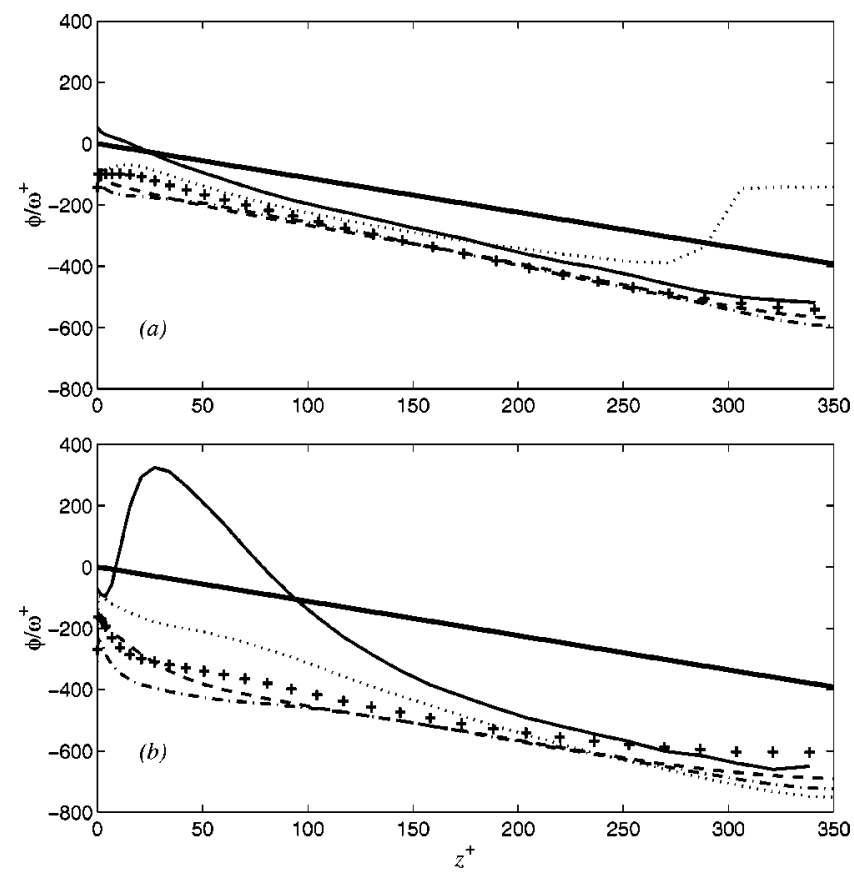

FIG. 12. Time lag (relative to the centerline velocity) of (-) shear; $(\cdots)$ $\left\langle u^{\prime \prime} u^{\prime \prime}\right\rangle ;(-\cdot-)\left\langle v^{\prime \prime} v^{\prime \prime}\right\rangle ;(--)\left\langle w^{\prime \prime} w^{\prime \prime}\right\rangle ;(+)\left\langle-u^{\prime \prime} w^{\prime \prime}\right\rangle$ vs distance from the wall, normalized with the forcing frequency. (a) $l_{s}^{+}=14$; (b) $l_{s}^{+}=35$. The thick line shows the slope predicted assuming a turbulent eddy diffusivity.

Sec. I; our calculations confirm the overall robustness of the mean turbulent quantities, and show that at very large driving frequencies $\left(l_{s}^{+}<5\right)$ the oscillating part decouples from the mean turbulent flow and tends toward the Stokes solution, while the amplitude of the oscillation in the turbulent quantities decreases. However, we refrain from claiming that our findings settle the above-mentioned controversy, since we need to determine the bursting and ejection frequency appropriate for our simulations in a self-consisting manner, namely by analyzing DNS (and LES) data in steady state conditions for the particular geometry and parameters considered in this study. Only in this way will we be able to determine whether $\omega^{+}=0.1$ lies in the very high-frequency limit.

While the meaning of $l_{t}^{+}$is best elucidated in the context of the time evolution of the flow (Sec. IV B), we can test the prediction of constant propagation speed by looking at the phase $\phi(z)$ of different turbulent quantities. A constant propagation speed requires that $\left(d \phi / d z^{+}\right) / \omega^{+}=-1 / c^{+}$. Figure 12 shows the time lag of the ac components of the Reynolds stress and the shear as a function of the distance from the wall. At intermediate frequencies, Fig. 12(a), all quantities show a linear dependence away from the generation area and up to $z^{+}=250$. The sharp change in the slope of the phase of $\left\langle u^{\prime \prime} u^{\prime \prime}\right\rangle$ marks the transition to "frozen" turbulence in the core. At low frequencies, Fig. 12(b), the phase relationship between the quantities considered is relatively constant away from the wall. Similar results hold for higher harmonics. These results confirm the notion that the eddy viscosity appropriate for the turbulent momentum transport (that is, $\nu_{t}=\kappa u_{\tau} z^{+}$) is also valid for the diffusion of turbulent kinetic energy.
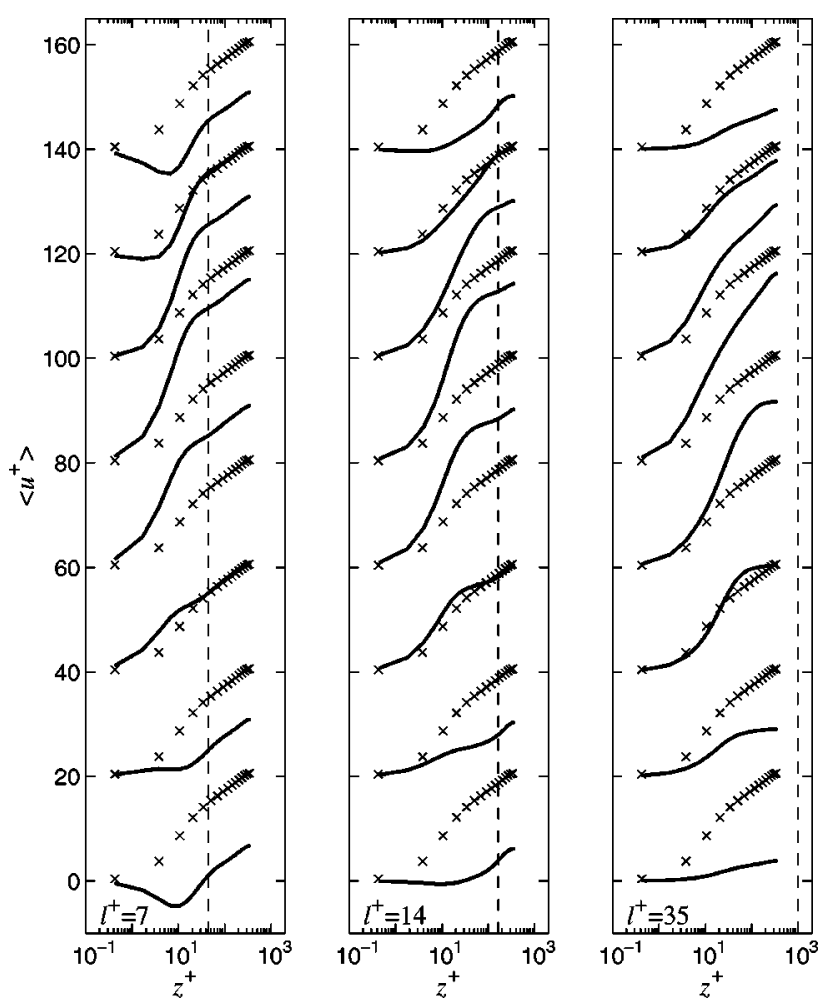

FIG. 13. Profiles of $\langle u\rangle$ at different phases of the wave cycle. Profiles are $T / 8$ apart and are offset by 20 units in the vertical direction. $(\times)$ Steady channel (every other point is shown). The dashed lines mark the position of $2 l_{t}^{+}$.

\section{B. Time evolution}

The robustness of the mean statistics even at low frequencies is all the more remarkable when one considers the detailed evolution of the flow within a wave cycle. In Fig. 13 we show the velocity profiles at eight equi-spaced phases during the cycle, starting at the beginning of the acceleration phase $(t / T=0 / 8)$. At high frequency the profile in the outer region $\left(z^{+}>2 l_{t}^{+}\right)$remains essentially unaltered and a logarithmic layer with the usual slope is present, which is shifted upwards and downwards by the modulation of the core velocity. At intermediate frequencies the profile undergoes several transformations, and a logarithmic layer is present in the outer layer only through part of the cycle, when the centerline velocity is near its maximum. Stronger coupling between the inner and outer layers occurs at this frequency. At low frequencies, $2 l_{t}$ is greater than the channel half-height and the entire flow is strongly affected by the oscillation. As will be shown later, the flow relaminarizes during the deceleration phase, and begins the acceleration phase with very little residual turbulent fluctuations. The retransition to turbulence that takes place during the acceleration phase results, eventually, in a velocity profile with a standard logarithmic layer. This, however, occurs only after the instabilities have had time to amplify and develop into "equilibrium" turbulent eddies, toward $t / T=5 / 8$ (the sixth profile from the bottom in Fig. 13). It is interesting to point out that, despite the crude nature of the underlying assumption, our estimate for $l_{t}^{+}$agrees reasonably well with the edge of the turbulent Stokes layer. 

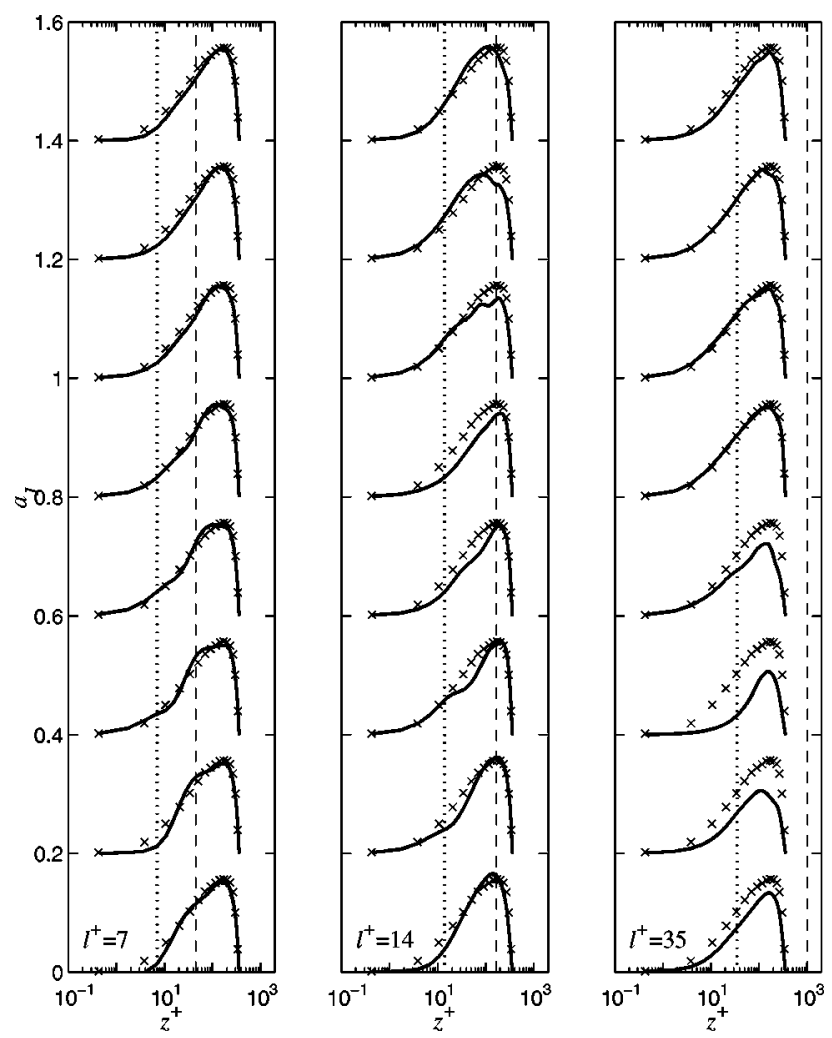

FIG. 14. Profiles of the structure parameter $a_{1}$ at different phases of the wave cycle. Profiles are $T / 8$ apart and are offset by 0.2 units in the vertical direction. $(X)$ Steady channel (every other point is shown). The dashed lines mark the position of $2 l_{t}^{+}$, the dotted lines the position of $l_{s}^{+}$.

The lack of a well-defined logarithmic layer at low and intermediate frequencies strongly suggest that the turbulence is far from equilibrium. To measure the departure from equilibrium in Fig. 14 we show the structure parameter $a_{1}$ $=-\left\langle u^{\prime \prime} w^{\prime \prime}\right\rangle\left\langle\left\langle u_{i}^{\prime \prime} u_{i}^{\prime \prime}\right\rangle\right.$, whose value, in the case of steady turbulence, is about 0.15 in the logarithmic layer. This quantity measures the efficiency with which the turbulent eddies extract Reynolds shear stress from a given amount of turbulent kinetic energy. Lower values of $a_{1}$ are observed in threedimensional boundary layers and nonequilibrium flows. For high driving frequencies very little difference is seen with respect to the steady case, and both inner and outer layers appear to be in near equilibrium. At the intermediate frequencies, on the other hand, lower values of $a_{1}$ can be observed near the interface $z^{+}=2 l_{t}^{+}$. These low values are initially generated near $z^{+} \simeq l_{s}^{+}$; during the cycle this region of deficit of $a_{1}$ extends and migrates toward the outer region. At low frequencies $a_{1}$ is below its equilibrium level during the acceleration stage (it actually decreases during the early part of the acceleration) and it returns to equilibrium values during the deceleration. So, while at intermediate frequencies the flow is never in equilibrium, at low frequencies the deceleration phase is in equilibrium. Figures 15 and 16 show, respectively, the trace of the Reynolds-stress tensor, $\left\langle q^{2}\right\rangle$ $=\left\langle u_{i}^{\prime \prime} u_{i}^{\prime \prime}\right\rangle$ (i.e., twice the turbulent kinetic energy), and the Reynolds shear stress, $\left\langle u^{\prime \prime} w^{\prime \prime}\right\rangle$. At high frequencies, for $z^{+}$ $>2 l_{t}^{+}$the turbulent kinetic energy (TKE) and the Reynolds stress are close to their values for steady channel flow. Some
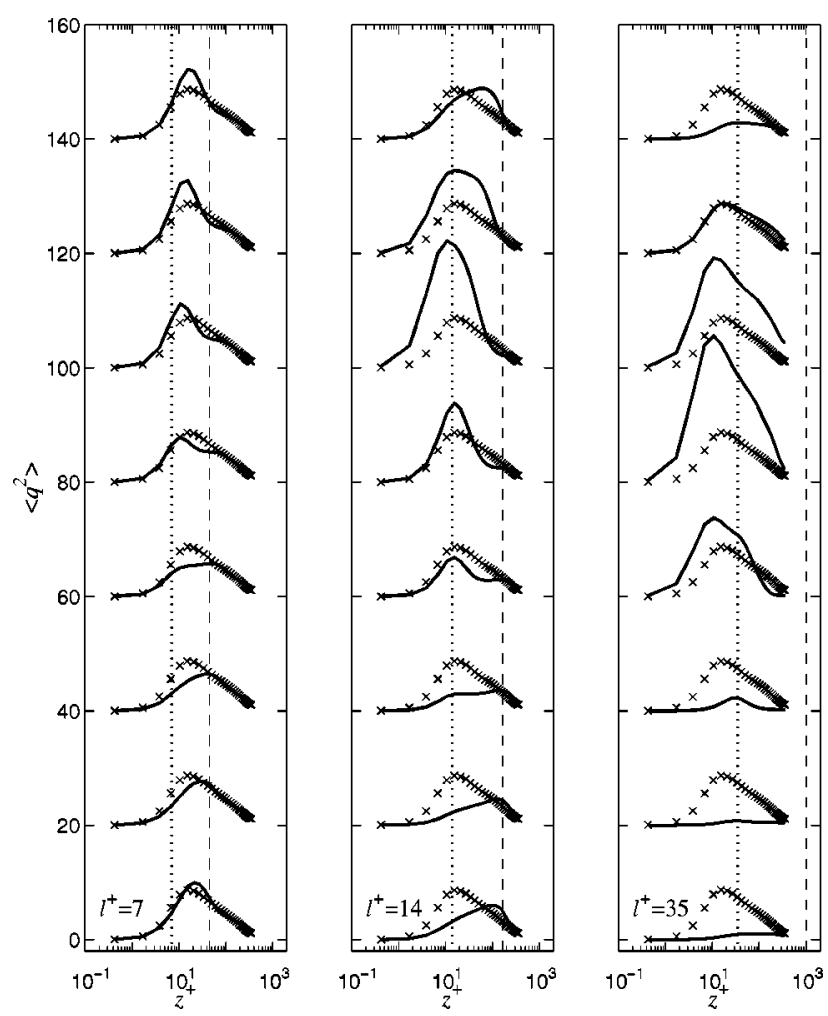

FIG. 15. Profiles of the trace of the Reynolds stress tensor, $\left\langle q^{2}\right\rangle$, at different phases of the wave cycle. Profiles are T/8 apart and are offset by 20 units in the vertical direction. $(\times)$ Steady channel. The dashed lines mark the position of $2 l_{t}^{+}$.

differences can be observed only in the inner layer. First, the position of the peak TKE oscillates through the cycle: during the acceleration phase it moves away; then a secondary peak is formed near $z^{+}>l_{s}^{+}$, which is rapidly amplified, and migrates away from the wall during the deceleration phase. A similar behavior can be observed in the shear stress, Fig. 16. The deficits of Reynolds stress and TKE are, however, proportional to each other, resulting in an unchanged value of $a_{1}$.

For the intermediate frequency, $l_{s}^{+}=14$, the double-peak behavior of the TKE is not observed: TKE is generated $z^{+}$ $\simeq l_{s}^{+}$during the acceleration phase and the peak slowly moves away from the wall. The growth of the TKE in the peak closer to the wall is accelerated. These effects may be due to the fact that in this case $l_{s}^{+}$is very close to the location of maximum production in the steady channel; thus, while at the high frequency two production mechanisms (the equilibrium production of TKE and an additional one due to the unsteadiness of the flow) are competing, here they are acting at the same location, and are hence amplifying each other. For $z^{+}>2 l_{t}^{+}$these quantities are not much different from the steady channel case.

Similar observations apply to the lower frequency, $l_{s}^{+}$ $=35$. However, a more intermittent behavior is observed: Significant TKE exists only for approximately $3 / 8$ of the cycle, the flow being nearly quiescent the rest of the time. This behavior suggests a relaminarization of the flow during the acceleration phase, followed by a sudden retransition. Note that although the level of turbulent fluctuations is dra- 

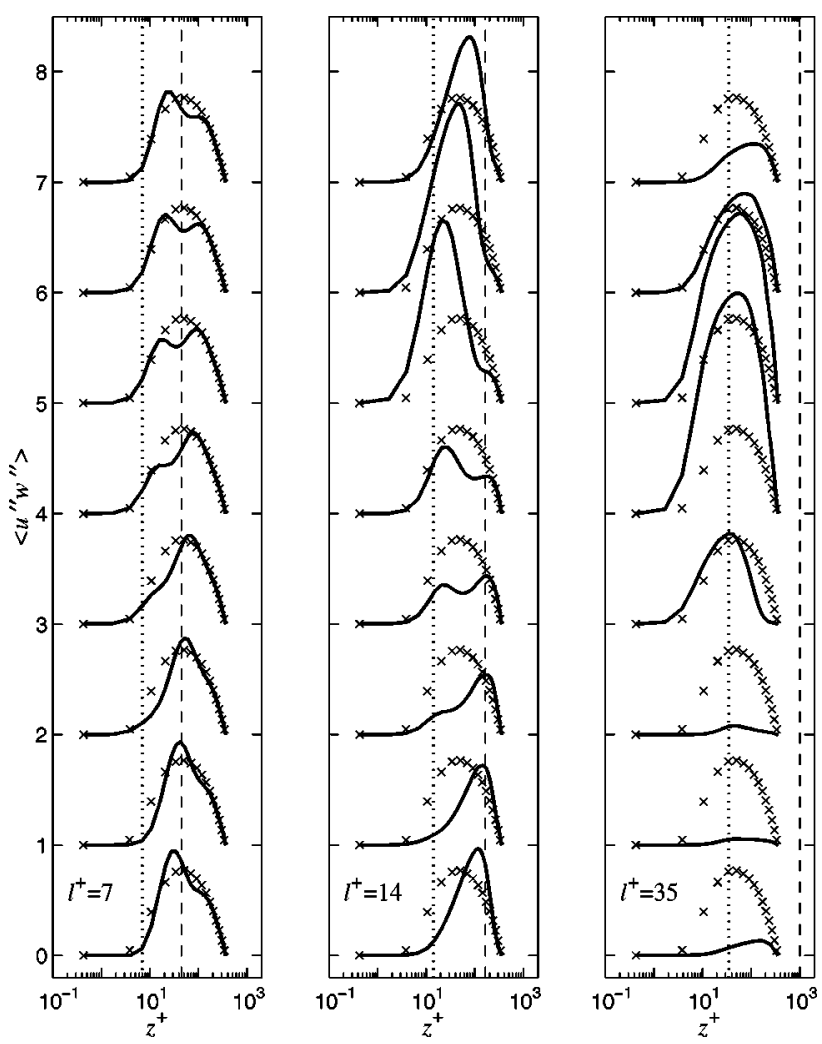

FIG. 16. Profiles of the Reynolds shear stress, $\left\langle u^{\prime \prime} w^{\prime \prime}\right\rangle$, at different phases of the wave cycle. Profiles are $T / 8$ apart and are offset by 2 units in the vertical direction. $(\times)$ Steady channel (every other point is shown). The dashed lines mark the position of $2 l_{t}^{+}$.

matically reduced, the mean velocity profile (and consequently the stress at the wall) is far from the laminar one, as the relaxation toward a laminar profile would take place on a time scale $t^{+}=\left(H^{2} / \nu\right) \times\left(u_{\tau}^{2} / \nu\right)=\left(\operatorname{Re}_{\tau}\right)^{2}$, which is much larger than the period of the forcing.

The Reynolds stress at high and intermediate frequencies shows quite well the wave-like structure of the time evolution in the inner layer, although the waves seem to penetrate further than $2 l_{t}^{+}$, unlike the other quantities that we have considered. At low frequencies we observe a pattern similar to the one for TKE.

The above-mentioned data were normalized using the time-averaged friction velocity. We investigated whether this strong modulation of the turbulent statistics could be taken into account by using an instantaneous normalization. However, neither the instantaneous friction velocity nor the centerline velocity collapsed the turbulent statistics, indicating that the modifications in the turbulence are not due directly to the forcing itself, but must be mediated by some more complex mechanism. This is to be expected given the departure from equilibrium found in $a_{1}$. Finally, it should be pointed out that the changes in $\left\langle q^{2}\right\rangle$ and $\left\langle u^{\prime \prime} w^{\prime \prime}\right\rangle$ are related, so that the changes in the structure coefficient are not nearly as dramatic as those in $\left\langle q^{2}\right\rangle$ and $\left\langle u^{\prime \prime} w^{\prime \prime}\right\rangle$. In particular, the behavior of $a_{1}$ at low frequencies show that the turbulence is in equilibrium during the decelerating part of the cycle, but strongly departs from it during the accelerating portion.

Figures 17-19 show the ratios of each of the normal stresses to their sum, $\left\langle q^{2}\right\rangle$. At the high frequency energy is
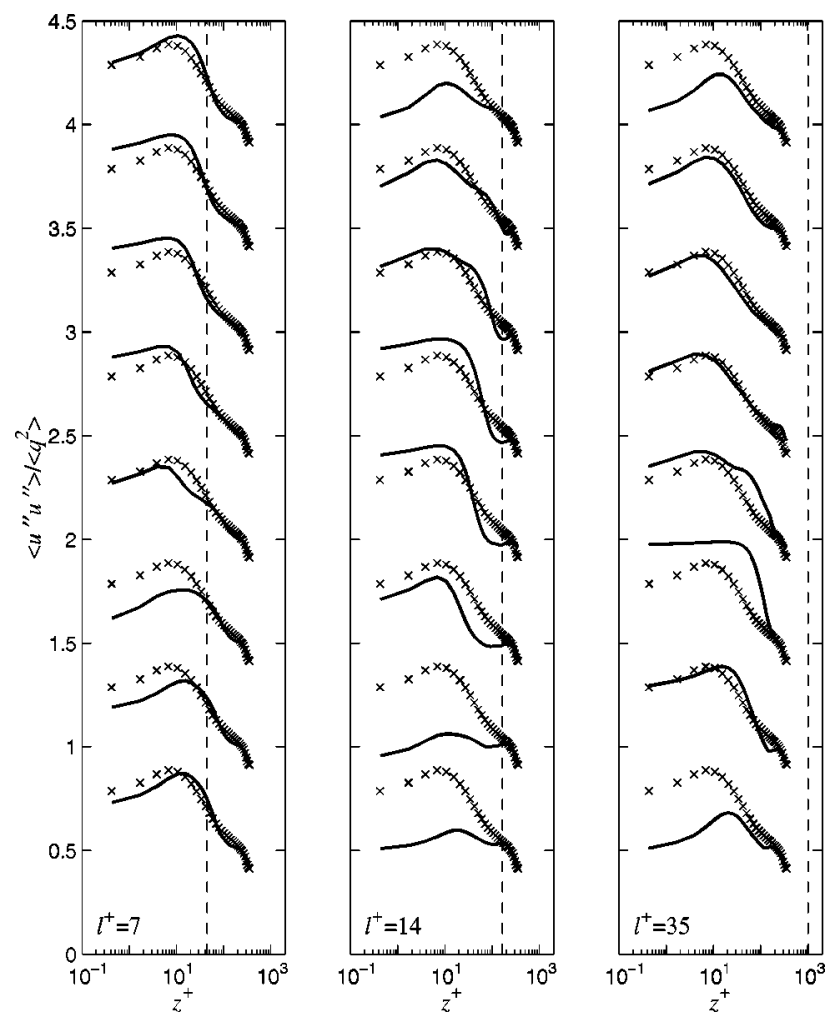

FIG. 17. Profiles of $\left\langle u^{\prime \prime} u^{\prime \prime}\right\rangle /\left\langle q^{2}\right\rangle$ at different phases of the wave cycle. Profiles are $T / 8$ apart and are offset by 0.5 units in the vertical direction. $(\times)$ Steady channel. The dashed lines mark the position of $2 l_{t}^{+}$.
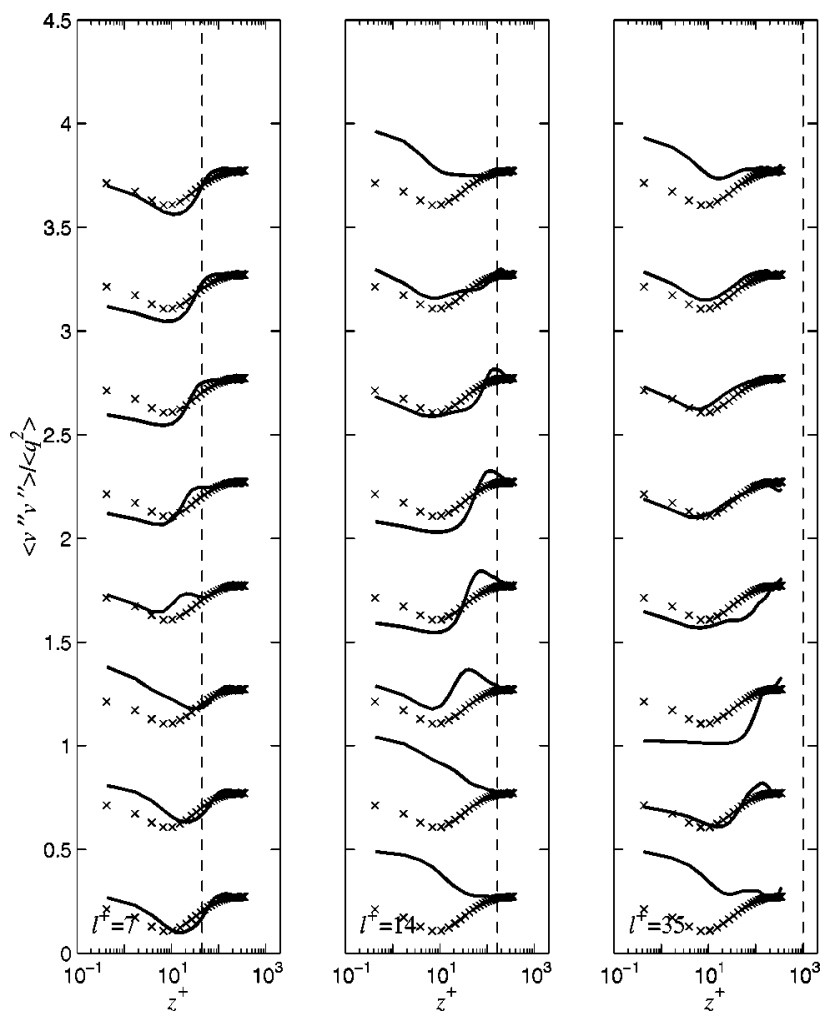

FIG. 18. Profiles of $\left\langle v^{\prime \prime} v^{\prime \prime}\right\rangle /\left\langle q^{2}\right\rangle$ at different phases of the wave cycle. Profiles are $T / 8$ apart and are offset by 0.5 units in the vertical direction. $(\times)$ Steady channel. The dashed lines mark the position of $2 l_{t}^{+}$. 

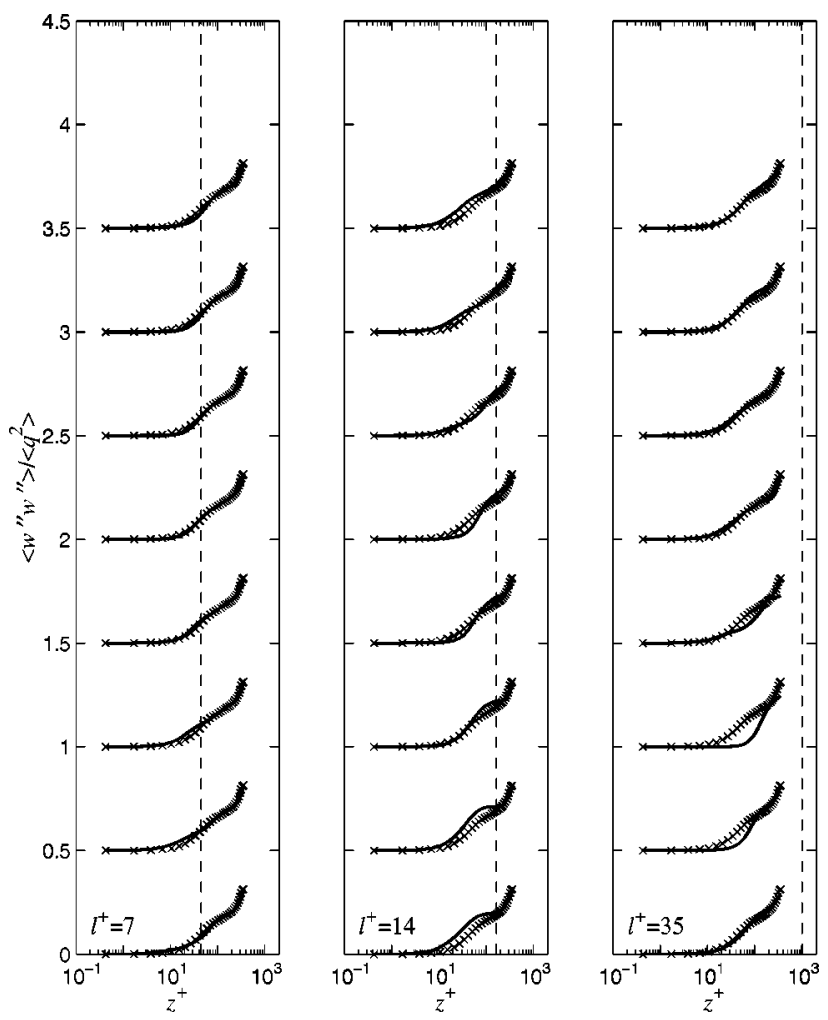

FIG. 19. Profiles of $\left\langle w^{\prime \prime} w^{\prime \prime}\right\rangle /\left\langle q^{2}\right\rangle$ at different phases of the wave cycle. Profiles are $T / 8$ apart and are offset by 0.5 units in the vertical direction. $(\times)$ Steady channel. The dashed lines mark the position of $2 l_{t}^{+}$.

seen to be exchanged between streamwise and spanwise fluctuations in the inner layer only. The spanwise fluctuations increase during the acceleration phase, decrease during the deceleration; the streamwise ones have the opposite behavior, while the wall-normal ones are not much affected. At moderate and low frequencies, this behavior is strongly amplified: The streamwise and spanwise fluctuations have approximately the same magnitude during the acceleration phase near the wall. This behavior indicates a tendency of the flow toward two dimensionality (in the sense that the streaks become more elongated and less wavy, as will be shown later) that is observed in various types of relaminarizing flows (Piomelli et al. ${ }^{55}$ ). Again, equilibrium is reached and maintained during the decelerating portion of the lowfrequency flow.

\section{Reynolds-stress budgets}

The behaviors described in Sec. IV B indicate that complex energy exchanges are taking place in the flow, possibly with a history effect. Also, notice that although the instantaneous Reynolds number oscillates between the same values, independent of the driving frequency, only the lowfrequency and quasisteady cases exhibit relaminarization (that is, the maximum turbulent kinetic energy is essentially zero during a significative portion of the cycle).

To elucidate the energy-producing mechanisms, the budgets of the resolved Reynolds stresses, $\left\langle u_{i}^{\prime \prime} u_{j}^{\prime \prime}\right\rangle$, were computed. In fully developed plane-channel flow the Reynoldsstress budgets can be written as
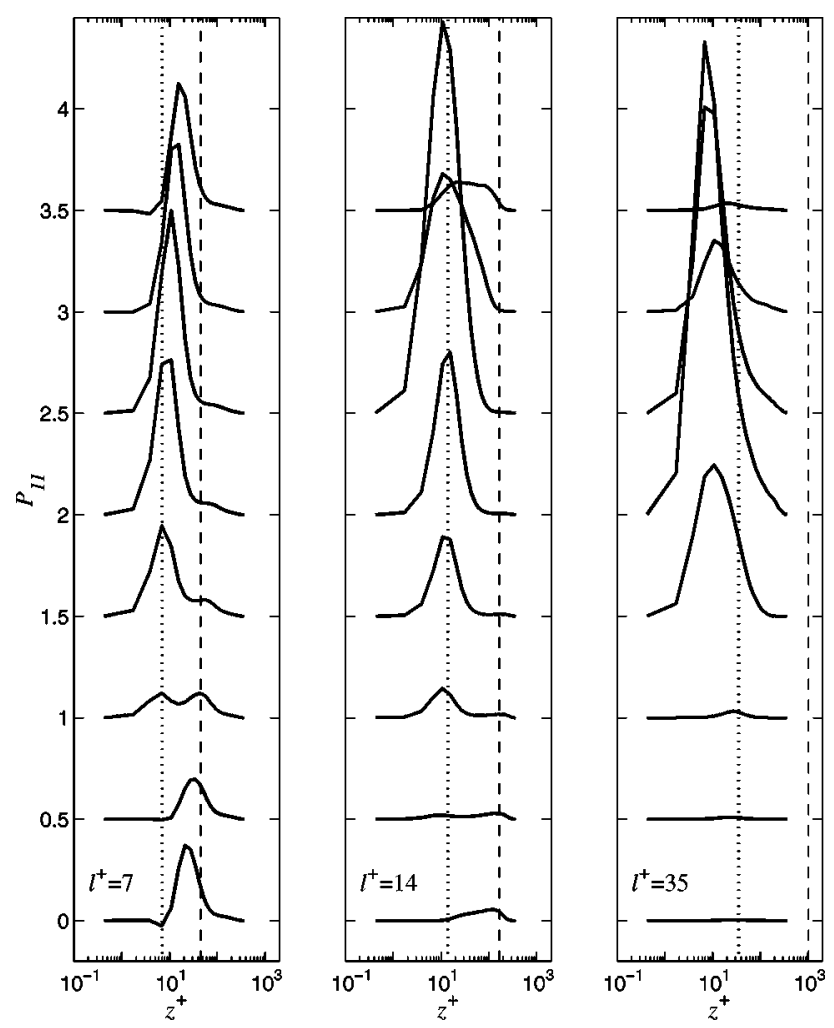

FIG. 20. Profiles of $P_{11}$ at different phases of the wave cycle. Profiles are $T / 8$ apart and are offset by 3 units in the vertical direction. The dashed lines mark the position of $2 l_{t}^{+}$, the dotted lines the position of $l_{s}^{+}$.

$$
\frac{\partial}{\partial t}\left\langle u_{i}^{\prime \prime} u_{j}^{\prime \prime}\right\rangle=P_{i j}+\Pi_{i j}+D_{i j}-\epsilon_{i j},
$$

where $P_{i j}, \Pi_{i j}, D_{i j}$, and $\epsilon_{i j}$ are, respectively, the production, velocity-pressure-gradient, diffusion (turbulent and viscous), and dissipation terms, which are given by

$$
\begin{aligned}
& P_{i j}=-\left\langle u_{i}^{\prime \prime} u_{3}^{\prime \prime}\right\rangle \frac{\partial\left\langle u_{j}\right\rangle}{\partial x_{3}}-\left\langle u_{j}^{\prime \prime} u_{3}^{\prime \prime}\right\rangle \frac{\partial\left\langle u_{i}\right\rangle}{\partial x_{3}} \\
& \Pi_{i j}=-\left\langle u_{i}^{\prime \prime} \frac{\partial p^{\prime \prime}}{\partial x_{j}}+u_{j}^{\prime \prime} \frac{\partial p^{\prime \prime}}{\partial x_{i}}\right\rangle \\
& D_{i j}=\frac{\partial}{\partial x_{3}}\left[\nu \frac{\partial}{\partial x_{3}}\left\langle u_{i}^{\prime \prime} u_{j}^{\prime \prime}\right\rangle-\left\langle u_{i}^{\prime \prime} u_{j}^{\prime \prime} u_{3}^{\prime \prime}\right\rangle+\left\langle\tau_{i 3} u_{j}^{\prime \prime}+\tau_{j 3} u_{i}^{\prime \prime}\right\rangle\right] \\
& \epsilon_{i j}=2 \nu\left\langle\frac{\partial u_{j}^{\prime \prime}}{\partial x_{k}} \frac{\partial u_{i}^{\prime \prime}}{\partial x_{k}}\right\rangle-\left\langle\tau_{i k} \frac{\partial u_{j}^{\prime \prime}}{\partial x_{k}}+\tau_{j k} \frac{\partial u_{i}^{\prime \prime}}{\partial x_{k}}\right\rangle
\end{aligned}
$$

For the flow we are considering, there is no advection, and the only nonzero components are the $11,22,33$, and 13 ones. Production affects only the 11 and 13 Reynolds stresses, while for the other components the major source of energy is the velocity-pressure-gradient term $\Pi_{i j}$.

At large driving frequencies the evolution of the production terms (Fig. 20) shows that the production is dominated by a wave that originates around $z^{+}=l_{s}^{+}$at $t=T / 4$, and moves outward, reaching its maximum at $t=5 / 8 T$. At lower frequencies, the origin of the wave can be traced to $t=T / 8$, and peaks at $t=5 / 8 T$. The outward-moving wave is less evi- 

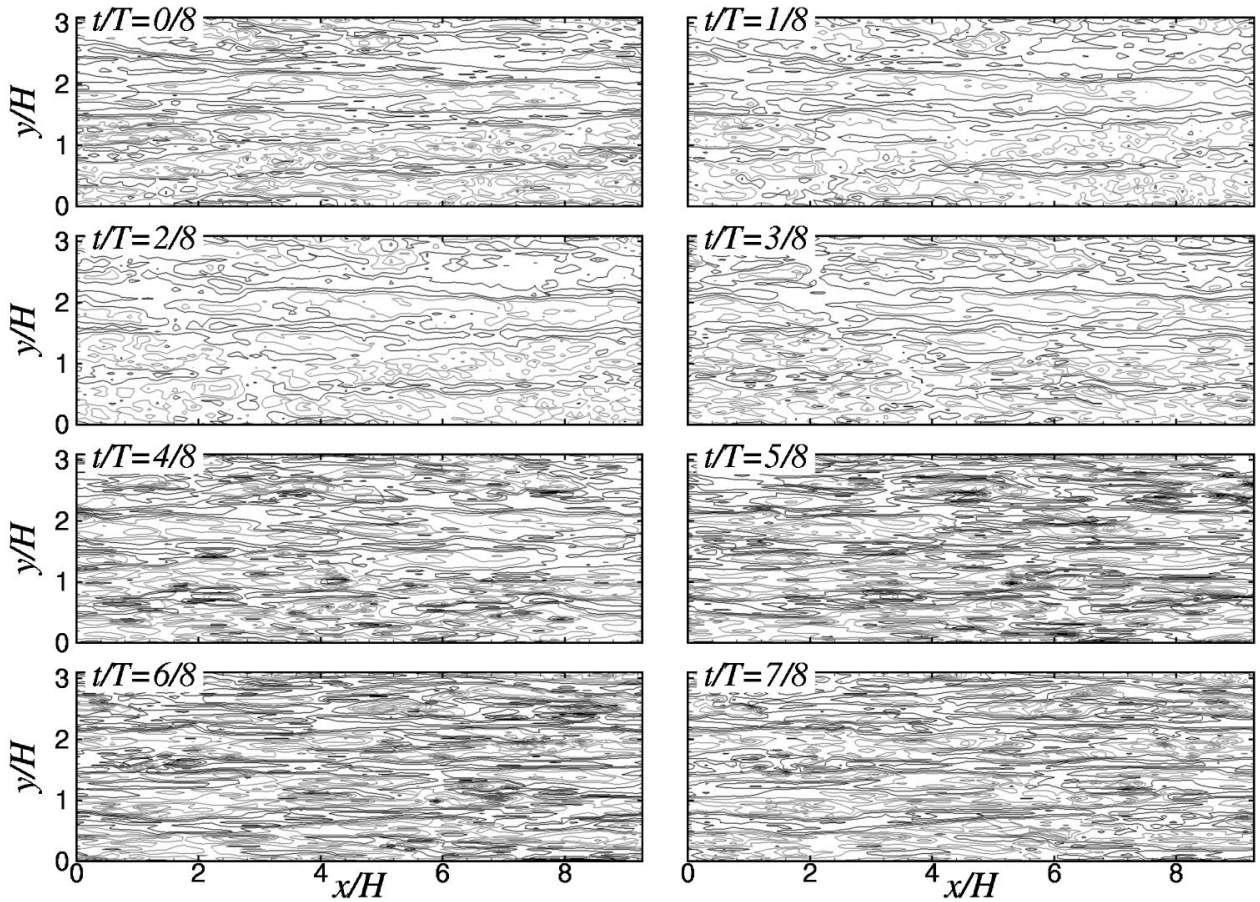

FIG. 21. Contours of the streamwise velocity fluctuations in the $z^{+}=10$ plane. $\operatorname{Re}_{s}=100$. Twelve equi-spaced contours between -11 and +11 ; negative contours in gray. dent but still present. At $l_{s}^{+}=35$ the wave seems to originate at the very beginning of the acceleration phase. Once production is restarted, the peak moves toward its usual location. The common feature is that $l_{s}^{+}$controls the position where the production wave is initiated. However, while at high and intermediate frequencies the flow remains turbulent during the whole length of the cycle, so that production is controlled by the modulation of the shear and the turbulent stress $\left\langle u^{\prime \prime} w^{\prime \prime}\right\rangle$, at low frequencies the flow essentially becomes fluctuation free at the end of the deceleration phase, so that turbulence must be restarted before the usual production mechanism can work. Interestingly, the phase averaged velocity profile satisfies the condition of the Fjortoft theorem $^{56}$ only during the last half of the deceleration cycle, when turbulence is dying. This issue will be further considered in Sec. IVD, when we will look at the instantaneous turbulent structures.

We observed that the evolution of $\left\langle q^{2}\right\rangle$ is driven almost entirely by changes in the production and dissipation. The diffusion terms (not shown) are important only at the beginning of the acceleration phase, for intermediate or high frequencies, and the velocity-pressure-gradient term is almost one order of magnitude smaller than these terms, and plays an important role only in the balance of $\left\langle v^{2}\right\rangle$ and $\left\langle w^{2}\right\rangle$. Finally, production correlates with the instantaneous Reynolds number only at low frequencies. At higher frequency the peak in production lags behind the peak in velocity. Also, at high frequency production never vanishes entirely. At intermediate frequencies, production is almost zero only briefly during the accelerating phase, but there is not enough time for turbulent dissipation to act, before production restarts. Only at low frequencies is there enough time to dissipate energy during deceleration, so that by the end of the decelerating phase the flow is essentially turbulence free.

\section{Turbulence structure}

The effect of the driving frequency on the turbulent statistics stems from a profoundly different topology of the turbulent structures. Also, it is very likely that the key to understanding the contradictory experimental and numerical results at very large driving frequencies is to be found in the way the oscillating pressure gradient alters the topology of the coherent structures. A detailed study of the problem is outside the scope of this paper. Here we briefly discuss some interesting features to support the conjectures made while discussing the time evolution of the turbulent quantities.

Even at high frequencies, the oscillating channel differs somewhat from the steady one. Figure 21 shows contours of the streamwise velocity fluctuations $u^{\prime \prime}$ during the cycle. After the velocity has reached its maximum, when the flow is in a fully turbulent regime $(4 / 8 \leqslant t / T \leqslant 7 / 8)$ fairly healthy streaky structures can be observed, even in the reversed-flow region in which this plane lies. During the acceleration phase the picture is different: some very long low-speed streaks can be observed in the initial stages $(2 / 8 \leqslant t / T \leqslant 3 / 8)$ consistent with the transfer of energy between the $u$ and $v$ components observed earlier. Also, the size of the streaks is larger. Although the plane under exam is inside the inner region $\left(z^{+}\right.$ $\left.=11<l_{t}^{+}\right)$, similar phenomena can be observed for $z^{+}$ $>l_{t}^{+}$, even at higher frequencies.

At intermediate frequency streaks are absent during the initial phase of the acceleration, although relatively strong fluctuations are present, and reappear around $t=T / 4$ (see the production plot in Fig. 20). At low frequencies the retransition process is very different: in Fig. 22 one can observe a nearly quiescent flow for $t / T=0$. As the flow picks up momentum, very long and smooth streaks develop which eventually become unstable and burst into a localized turbulent 

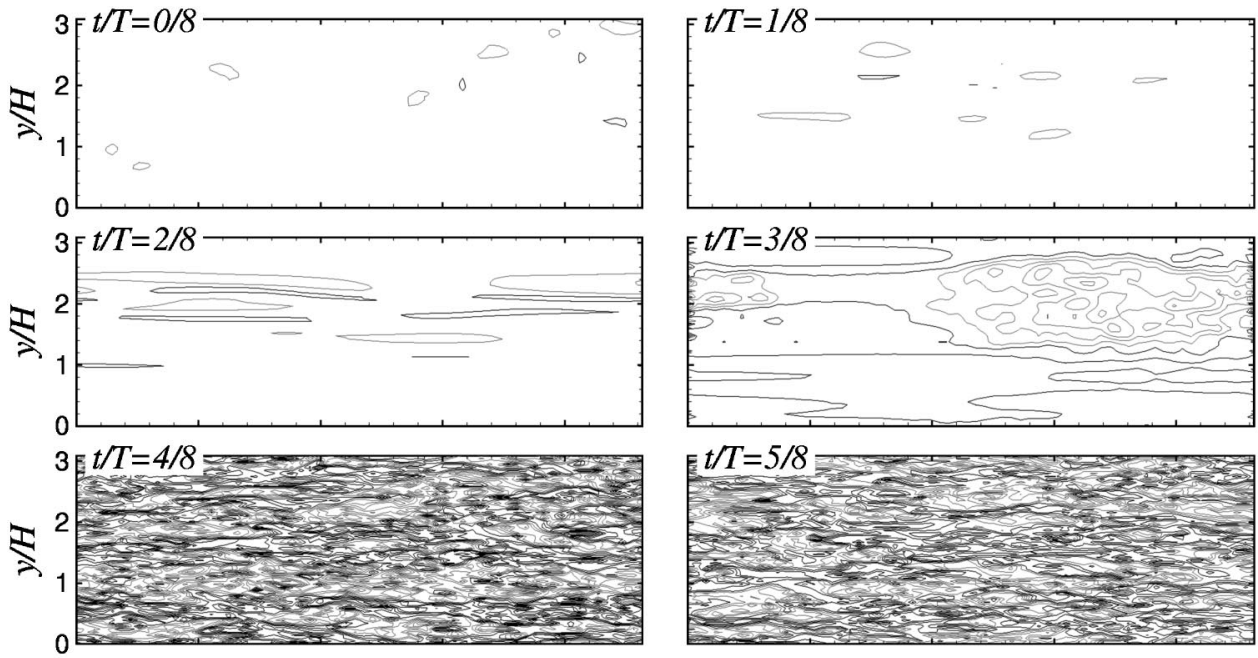

FIG. 22. Contours of the streamwise velocity fluctuations in the $z^{+}=10$ plane. $\operatorname{Re}_{s}=500$. Twelve equi-spaced contours between -11 and +11 ; negative contours in gray.
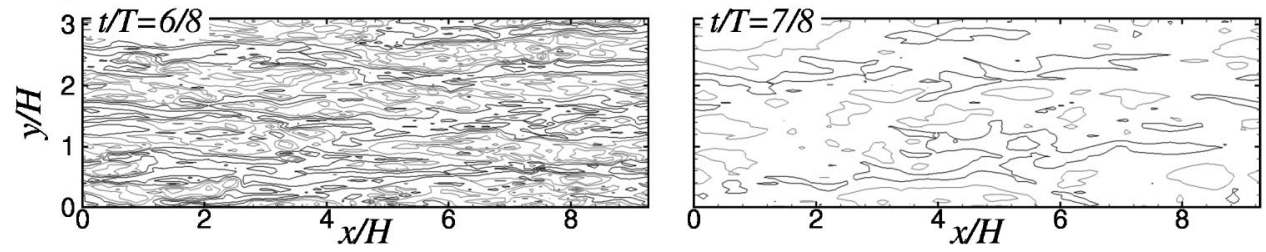

spot, at $t=T / 4$, which eventually fills the whole channel. Very long and smooth streaks undergoing a spot-like transition have been also reported by $\operatorname{Sarpkaya}^{1}$ (see his Fig. 7), even though in his experiments there was no mean flow. If the amplitude of the oscillation is large enough that relaminarization takes place, it is likely that the transition to turbulence is controlled more by the rate of acceleration than by the absolute velocity, since relaminarization "erases" the turbulent "memory" of the system.

In Fig. 23 the iso-surfaces of $Q$ are superposed on contours of $u^{\prime \prime}$ in a near-wall plane. $Q$ is defined as

$$
Q=-\frac{1}{2} \frac{\partial u_{i}^{\prime \prime}}{\partial x_{j}^{\prime \prime}} \frac{\partial u_{i}^{\prime \prime}}{\partial x_{j}^{\prime \prime}}=-\frac{1}{2}\left(S_{i j}^{\prime \prime} S_{i j}^{\prime \prime}-\Omega_{i j}^{\prime \prime} \Omega_{i j}^{\prime \prime}\right),
$$

where $S_{i j}^{\prime \prime}$ and $\Omega_{i j}^{\prime \prime}$ are, respectively, the symmetric and antisymmetric parts of the fluctuating velocity-gradient tensor (i.e., the strain rate and the rotation tensors). $Q$ has been found $^{57}$ to be an effective way to visualize the regions of coherent vorticity due to rotational motion (as opposed to those due to shear). The coherent eddies at this stage are significantly more disorganized, whereas during the turbulent phase (Fig. 24) a more canonical structure can be observed with quasistreamwise vortices near the wall and arches further away from it.

\section{CONCLUSIONS}

In this paper we studied numerically the flow in a turbulent channel subject to unsteady pressure gradient. The parameters used span the whole range of significant frequencies for a given ratio of oscillating-to-steady centerline velocity. The goals were (i) to investigate the appropriateness of using LES in unsteady flows and (ii) to characterize the physics of the flows. The first task was motivated by the fact that the turbulence in the frequency range considered is not in equi- librium. The comparison was done a posteriori using results from a DNS at $\operatorname{Re}_{s}=100$, as well as comparing the results with the experiments of the Grenoble and Iowa groups. The agreement was quite good in both cases. The mean properties, averages over many periods, were found to be relatively insensitive to the forcing frequencies, in agreement with several experimental results.

The study of the phase-averaged quantities showed that their dynamics are characterized by waves that originate in the viscous and buffer layers and propagate away from the wall. The generation area seemed to be centered around $l_{s}^{+}$, during the acceleration phase.

In order to study the propagation/attenuation process, following $\mathrm{Tu}$ and $\operatorname{Ramaprian}^{20}$ we introduced a length $l_{t}$, which measures how far the oscillations generated near the wall penetrate into the flow. If $2 l_{t}$ is significantly smaller than the half-height of the channel $H$, the core flow and the Stokes flow are not strongly coupled. Since $l_{t}$ is inversely related to the frequency, the system effectively acts as a lowpass filter. High-frequency oscillations are damped before reaching the logarithmic layer, with the cutoff frequency proportional to the mean turbulent intensity. In our calculations this was the case for the two largest driving frequencies considered. At low frequencies $2 l_{t}$ was larger than $H$ and a quite different flow was observed. At very high frequencies the fluctuations were confined within the viscous sublayer, and the inner flow was completely decoupled. At the second highest frequency that we considered the fluctuations were for the most part restricted to the viscous and buffer layers. In these layers the structure coefficients departed from the equilibrium (mean) value. The departure from nonequilibrium was more pronounced for the intermediate-frequency case, while at low frequency the flow was at equilibrium during the decelerating phase, but far from it, actually in a 


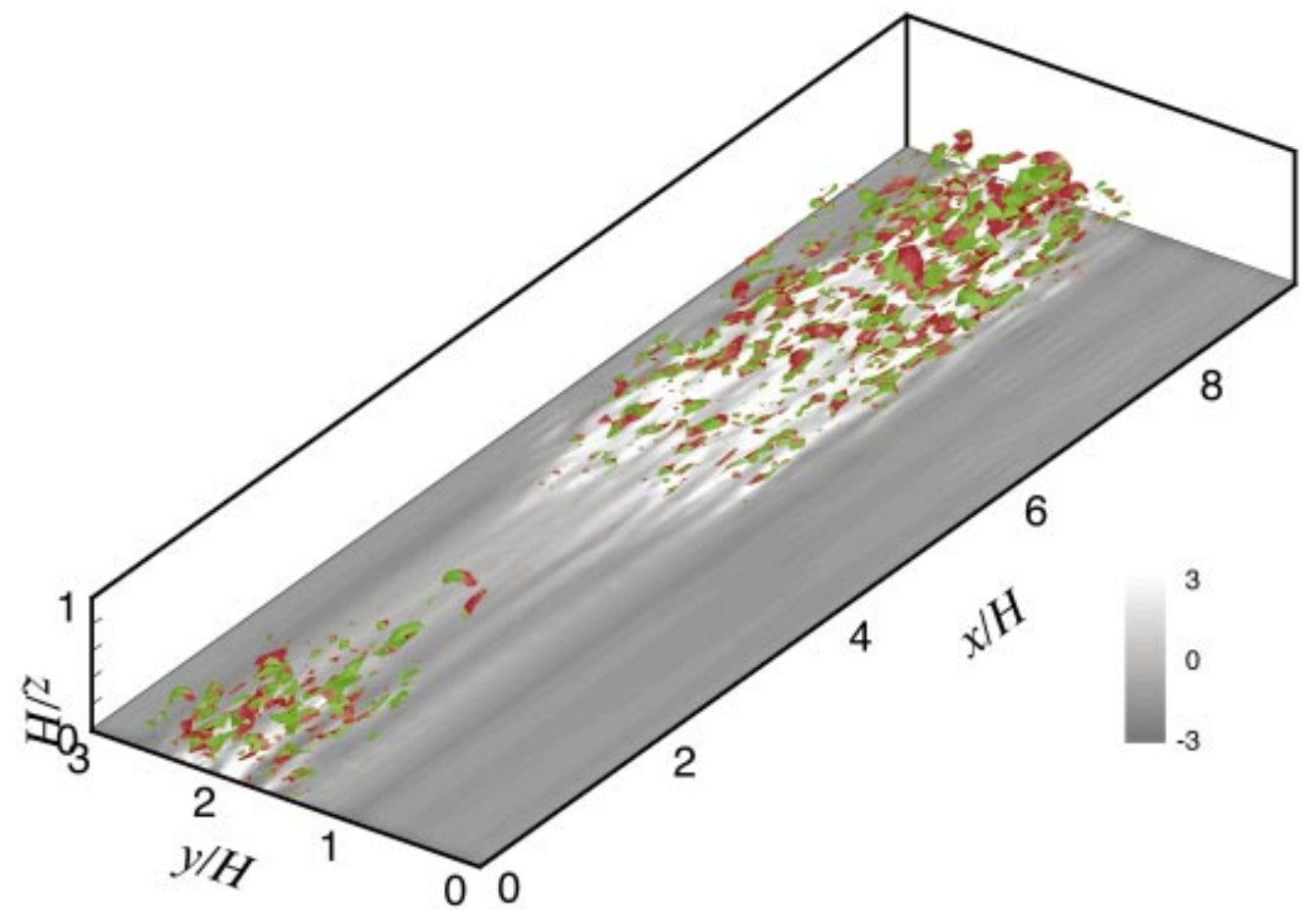

FIG. 23. (Color) Iso-surfaces of $Q=200 u_{\tau}^{2} / H^{2}$ superimposed on the streamwise velocity fluctuation contours in the $z^{+}=4$ plane. $\mathrm{Re}_{s}=500, t / T=3 / 8$. Regions associated with positive streamwise vorticity fluctuations are in green, negative in red.

quasilaminar state, during the acceleration phase, throughout the channel.

The scenario observed was that fluctuations begin to appear during the acceleration phase around $z^{+}=l_{s}^{+}$, undergo a rapid growth, and subsequently propagate outward as an attenuated wave, with a speed given by $\omega^{+} l_{t}^{+}$. It was noted that away from the generation region the phase difference between different turbulent quantities remained roughly constant, while in the generation region a more complicate behavior, dependent of the driving frequency, was observed.

Lastly, we have looked at the coherent structures observed in the flows. Unlike the mean properties, turbulent

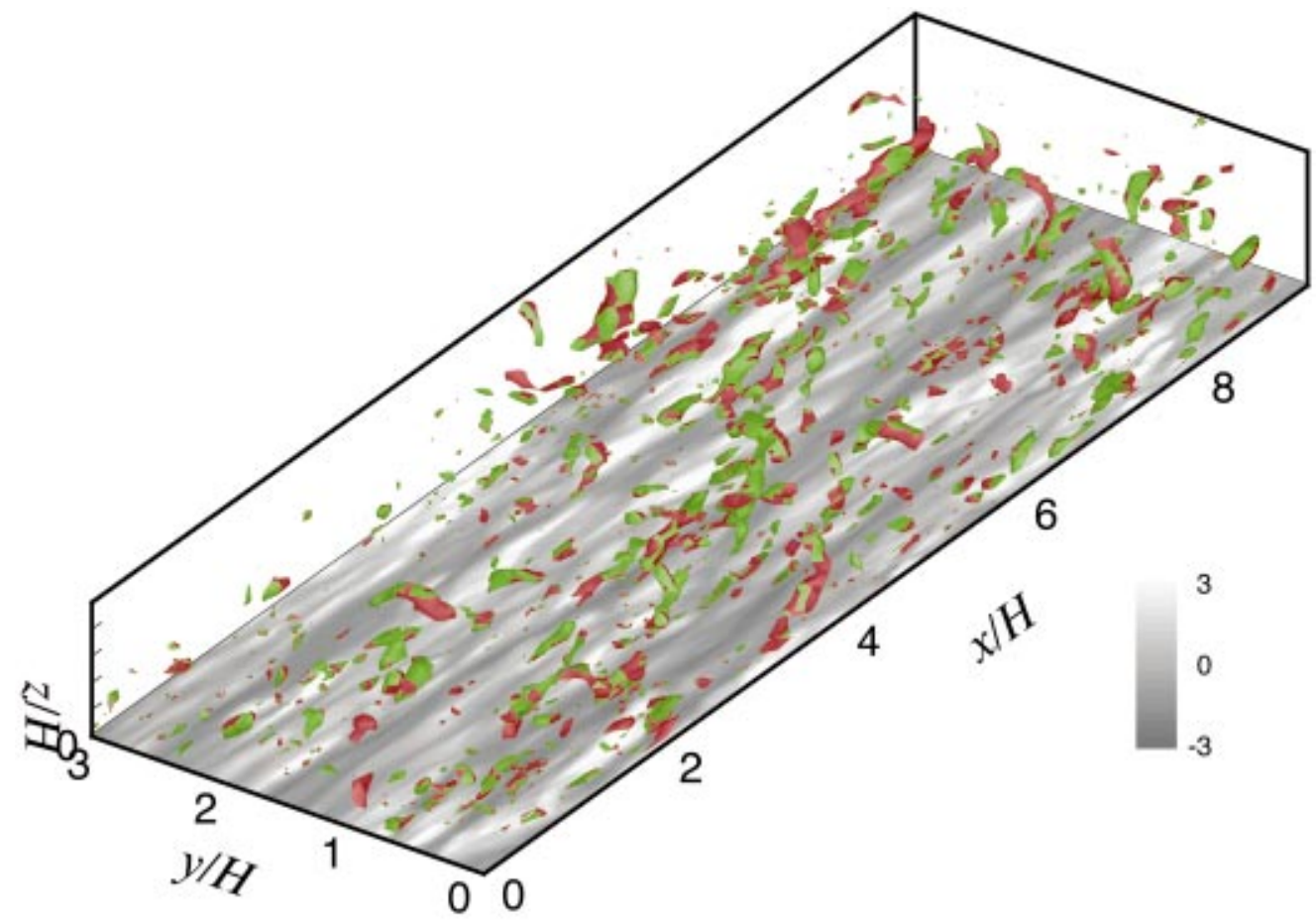

FIG. 24. (Color) Iso-surfaces of $Q=200 u_{\tau}^{2} / H^{2}$ superimposed on the streamwise velocity fluctuation contours in the $z^{+}=4$ plane. $\mathrm{Re}_{s}=500, t / T=6 / 8$. Regions associated with positive streamwise vorticity fluctuations are in green, negative in red. 
structures react quite differently depending on the driving frequency. The density and distribution of coherent structures was observed to be different from the steady case, and phase dependent. The picture was particularly interesting at low frequency. The flow begins the acceleration phase with very little turbulent kinetic energy. As it accelerates, long, smooth streaks emerge. During the last half of the acceleration stage a turbulent spot emerges, which spreads to the entire flow by the time the deceleration phase begins.

Several outstanding questions remain. Why are the time averaged quantities so robust, in spite of the rich diversity of behavior found when the time evolution is considered? How well can RANS model predict these flows? Why is the production wave generated at $z^{+}=l_{s}^{+}$? What is the instability mechanism behind the retransition at low frequencies? In the very high-frequency regime, does resonance with the bursting mechanism take place? Some of these issues are the focus of ongoing research.

\section{ACKNOWLEDGMENTS}

This work was initiated when the first author was a Postdoctoral Scholar at the Woods Hole Oceanographic Institution, with funding provided by the Johnson Foundation. Part of the work was supported by NSF Grant No. OCE-9910883. Computations were performed using resources made available by NCSA at the University of Illinois at UrbanaChampaign and by ATN at the University of North Carolina at Chapel Hill. Their support is gratefully acknowledged.

${ }^{1}$ T. Sarpkaya, "Coherent structures in oscillatory boundary layers," J. Fluid Mech. 253, 105 (1993).

${ }^{2}$ S. Tardu and G. Binder, "Reaction of bursting to an oscillating homogeneous pressure gradient," Eur. J. Mech. B/Fluids 16, 89 (1997).

${ }^{3}$ G. G. Stokes, "'On the effect of the internal friction of fluids on the motion of pendulums," Trans. Cambridge Philos. Soc. 9, 8 (1850); reprinted in G. G. Stokes, Mathematical and Physical Papers (Cambridge University Press, Cambridge, 1901).

${ }^{4}$ J. R. Womersley, "Method for the calculation of velocity rate of flow and viscous drag in arteries when the pressure gradient is known," J. Physiol. (London) 127, 553 (1955).

${ }^{5} \mathrm{~S}$. Uchida, "The pulsating viscous flow superimposed on the steady laminar motion of incompressible fluid in a circular pipe,' Z. Angew. Math. Phys. 7, 377 (1956).

${ }^{6}$ M. Y. Gündoğdu and M. Ö. Çarpinlioğlu, "Present state of art on pulsatile flow theory. Laminar and transitional flow regimes,' JSME Int. J., Ser. B 42, 384 (1999).

${ }^{7}$ M. Y. Gündoğdu and M. Ö. Çarpinlioğlu, "Present state of art on pulsatile flow theory. 2. Turbulent flow regime," JSME Int. J., Ser. B 42, 398 (1999).

${ }^{8}$ I. G. Jonsson, "Wave boundary layers and friction factors," Proceedings of the Tenth Coastal Engineering Conference 1966, Vol. 1, pp. 127-148.

${ }^{9}$ M. Hino, M. Kashiwayanagi, A. Nakayama, and T. Hara, "Experiments on the turbulence statistics and the structure of a reciprocating oscillatory flow," J. Fluid Mech. 131, 63 (1983).

${ }^{10}$ B. L. Jensen, B. M. Sumer, and J. Fredsœ, "Turbulent oscillatory boundary layers at high Reynolds numbers," J. Fluid Mech. 206, 265 (1989).

${ }^{11}$ R. Akhavan, R. D. Kamm, and A. H. Shapiro, "An investigation of transition to turbulence in bounded oscillatory Stokes flows. Part 1. Experiments,' J. Fluid Mech. 225, 395 (1991).

${ }^{12}$ C. R. Lodahl, B. M. Sumer, and J. Fredsœ, "'Turbulent combined oscillatory flow and current in a pipe,'” J. Fluid Mech. 373, 313 (1998).

${ }^{13}$ P. R. Spalart and B. S. Baldwin, "Direct simulation of a turbulent oscillating boundary layer," in Turbulent Shear Flows 6 (Springer, Berlin, 1987).

${ }^{14}$ R. Akhavan, R. D. Kamm, and A. H. Shapiro, "An investigation of tran- sition to turbulence in bounded oscillatory Stokes flows. 2. Numerical simulations,", J. Fluid Mech. 225, 423 (1991).

${ }^{15}$ W. D. Grant and O. S. Madsen, "The continental-shelf bottom boundary layer,’' Annu. Rev. Fluid Mech. 18, 265 (1986).

${ }^{16}$ The plus sign denotes quantities normalized using the kinematic viscosity $\nu$ and the mean friction velocity, $u_{\tau}=\sqrt{H \Delta P_{o} / 2 \rho L_{x}}$, where $H$ is the channel half-height and $\Delta P_{0} / L_{x}$ the mean pressure gradient.

${ }^{17} \mathrm{~S}$. Tardu and G. Binder, "Wall shear stress modulation in unsteady turbulent channel flow with high imposed frequencies," Phys. Fluids A 5, 2028 (1993).

${ }^{18}$ S. Tardu, G. Binder, and R. F. Blackwelder, "Turbulent channel flow with large-amplitude velocity oscillations,'” J. Fluid Mech. 267, 109 (1994).

${ }^{19}$ G. Binder, S. Tardu, and P. Vezin, "Cyclic modulation of Reynolds stresses and length scales in pulsed turbulent channel flow," Proc. R. Soc. London, Ser. A 451, 121 (1995).

${ }^{20}$ S. W. Tu and B. R. Ramaprian, "Fully developed periodic turbulent pipe flow. Part 1. Main experimental results and comparison with predictions," J. Fluid Mech. 137, 31 (1983).

${ }^{21}$ B. R. Ramaprian and S. W. Tu, "Fully developed periodic turbulent pipe flow. Part 2. The detailed structure of the flow,"' J. Fluid Mech. 137, 59 (1983).

${ }^{22}$ Z.-X. Mao and T. J. Hanratty, "Studies of the wall shear stress in a turbulent pulsating pipe flow," J. Fluid Mech. 170, 545 (1986).

${ }^{23}$ D. S. Finnicum and T. J. Hanratty, "Influence of imposed flow oscillations on turbulence,"' PhysicoChem. Hydrodyn. 10, 585 (1988).

${ }^{24}$ Z. Mao and T. J. Hanratty, "Influence of large-amplitude oscillations on turbulent drag," AIChE J. 40, 1601 (1994).

${ }^{25}$ G. J. Brereton, W. C. Reynolds, and R. Jayaraman, "Response of a turbulent boundary layer to sinusoidal free-stream unsteadiness," J. Fluid Mech. 221, 131 (1990).

${ }^{26} \mathrm{G}$. J. Brereton and W. C. Reynolds, "Dynamic response of boundary-layer turbulence to oscillatory shear," Phys. Fluids A 3, 178 (1991).

${ }^{27}$ This quantity, inversely related to the driving frequency, seems to have been rediscovered by several authors. To the best of our knowledge it was introduced first by Tu and Ramaprian (Ref. 20). Below we give a different (hopefully physically more transparent) derivation.

${ }^{28}$ J.-L. Hwang and G. J. Brereton, "Turbulence in high-frequency periodic fully-developed pipe flow," in Turbulent Shear Flows 8, selected papers from the Eighth International Symposium on Turbulent Shear Flows, Munich, Germany, 9-11 September, 1991, edited by F. Durst, R. Friedrich, B. E. Launder, F. W. Schmidt, U. Schuman, and J. H. Whitelaw (Springer, Berlin, 1993).

${ }^{29}$ J. Kim and P. R. Spalart, "Scaling of the bursting frequency in turbulent boundary layers at low Reynolds numbers," Phys. Fluids 30, 3326 (1987).

${ }^{30}$ S. Demare, L. Labraga, and C. Tournier, "Comparison and scaling of the bursting period in rough and smooth walls channel flows," J. Fluids Eng. 121, 735 (1999).

${ }^{31}$ T. S. Luchik and W. G. Tiederman, "Timescale and structure of ejections and bursts in turbulent channel flow," J. Fluid Mech. 174, 529 (1987).

${ }^{32} \mathrm{~T}$. Wei and W. W. Willmarth, "Reynolds-number effects on the structure of a turbulent channel flow,' J. Fluid Mech. 204, 57 (1989).

${ }^{33} \mathrm{~K}$. Hanjalic et al. "Computation of oscillating turbulent lows at transitional Re numbers,' Ninth International Symposium of Turbulent Shear Flows, Kyoto, 1993.

${ }^{34}$ K. Hanjalic, I. Hadzic, and S. Jakirlic, "Modeling turbulent wall flows subjected to strong pressure variations," J. Fluids Eng. 121, 57 (1999).

${ }^{35}$ R. R. Mankbadi and J. T. C. Liu, "Near-wall response in turbulent shear flows subjected to imposed unsteadiness,"' J. Fluid Mech. 238, 55 (1992).

${ }^{36}$ J. Reichardt, "Die Grundlagen des turbulenten Wärmeüberganges," Arch. Ges. Warmetechnik 2, 129 (1951).

${ }^{37}$ E. R. Van Driest, "'On the turbulent flow near a wall,'” J. Aerosp. Sci. 23, 1007 (1956)

${ }^{38}$ A. Scotti and U. Piomelli, "Turbulence models in pulsating flows," AIAA Paper No. 2001-0729 (2001).

${ }^{39}$ J. Kim, P. Moin, and R. D. Moser, "Turbulence statistics in fullydeveloped channel flow at low Reynolds number," J. Fluid Mech. 177, 133 (1987).

${ }^{40} \mathrm{P}$. R. Spalart, "Direct simulation of a turbulent boundary layer up to $\operatorname{Re}_{\theta}=1410$,' J. Fluid Mech. 187, 61 (1988).

${ }^{41}$ S. K. Robinson, "The kinematics of turbulent boundary layer structure," NASA Tech. Memo 103859 (1991).

${ }^{42}$ C.-T. Hsu, X. Lu, and M.-K. Kwan, "LES and RANS studies of oscillating flows over flat plate,'” ASCE, J. Eng. Mech. 126, 186 (2000).

${ }^{43}$ M. Germano, U. Piomelli, P. Moin, and W. H. Cabot, "A dynamic 
subgrid-scale eddy viscosity model,"' Phys. Fluids A 3, 1760 (1991).

${ }^{44}$ P. G. Saffman, "A model for inhomogeneous turbulent flow," Proc. R. Soc. London, Ser. A 317, 417 (1970)

${ }^{45}$ A. Yakhot, M. Arad, and G. Ben-Dor, "Numerical investigation of a laminar pulsating flow in a rectangular duct," Int. J. Numer. Methods Fluids 29, 935 (1999).

${ }^{46} \mathrm{~S}$. Rida and K. Dan Tran, "Direct simulation of turbulent pulsed plane channel flow," Eighth Symposium on Turbulent Shear Flows, Munich, Germany, 9-11 September, 1991.

${ }^{47}$ D. K. Lilly, "A proposed modification of the Germano subgrid-scale closure method," Phys. Fluids A 4, 633 (1992).

${ }^{48}$ U. Piomelli, "High Reynolds number calculations using the dynamic subgrid-scale stress model," Phys. Fluids A 5, 1484 (1993).

${ }^{49}$ T. A. Zang and M. Y. Hussaini, "Numerical experiments on subcritical transition mechanisms," AIAA Paper 85-0296 (1985).

${ }^{50}$ T. A. Zang and S. E. Krist, "Numerical experiments on stability and transition in plane channel flow," Theor. Comput. Fluid Dyn. 1, 41 (1989)
${ }^{51}$ U. Piomelli, A. Scotti, and E. Balaras, "Large-eddy simulation: From desktop to supercomputer," in Proceedings VECPAR2000, edited by J. Laginha Palma and J. Dongarra (Springer, Heidelberg, 2001).

${ }^{52}$ U. Piomelli and T. A. Zang, "Large-eddy simulation of transitional channel flow," Comput. Phys. Commun. 65, 224 (1991).

${ }^{53}$ D. Ronneberger and C. D. Ahrens, "Wall shear stress caused by signal amplitude perturbations of turbulent boundary layer flow: An experimental investigation," J. Fluid Mech. 83, 433 (1977).

${ }^{54} \mathrm{Z}$. Mao and T. J. Hanratty, "Measurement of wall shear rate in large amplitude unsteady reversing flows," Exp. Fluids 12, 342 (1992).

${ }^{55}$ U. Piomelli, E. Balaras, and A. Pascarelli, "Coherent structures in accelerating boundary layers," J. Turbulence 1, 1 (2000).

${ }^{56} \mathrm{R}$. Fjortoft, "Application of integral theorems in deriving criteria of stability for laminar flows and for the baroclinic circular vortex," Geofys. Publ., Oslo 17, 1 (1950).

${ }^{57}$ J. C. R. Hunt, A. A. Wray, and P. Moin, "Eddies, streams and convergence zones in turbulent flows," Proceedings of the Summer Program 1988, Center for Turbulence Research, 1988, p. 193. 